CERN-EP/2003-006

20 January 2003

\title{
Investigation of $K_{\mathrm{L}, \mathrm{S}} \rightarrow \pi^{+} \pi^{-} e^{+} e^{-}$decays
}

\begin{abstract}
NA48 Collaboration
A. Lai, D. Marras

Dipartimento di Fisica dell'Università e Sezione dell'INFN di Cagliari, I-09100 Cagliari, Italy.
\end{abstract}

A. Bevan ${ }^{1)}$, R.S. Dosanjh, T.J. Gershon ${ }^{2)}$, B. Hay ${ }^{3)}$, G.E. Kalmus, C. Lazzeroni, D.J. Munday, M.D. Needham ${ }^{4}$, E. Olaiya, M.A. Parker, T.O. White, S.A. Wotton Cavendish Laboratory, University of Cambridge, Cambridge, CB3 OHE, U.K. ${ }^{5}$.

G. Barr, G. Bocquet, A. Ceccucci, D. Cundy, N. Doble, V. Falaleev, L. Gatignon, A. Gonidec, B. Gorini, G. Govi, P. Grafström, W. Kubischta, M. Lenti' ${ }^{6)}$, S. Luitz ${ }^{7)}$, I. Mikulec ${ }^{8)}$, A. Norton, S. Palestini, B. Panzer-Steindel, H. Taureg, M. Velasco, H. Wahl CERN, CH-1211 Genève 23, Switzerland.

C. Cheshkov, A. Gaponenko ${ }^{9)}$, P. Hristov, V. Kekelidze, D. Madigojine, G. Tatishviliin), Yu. Potrebenikov, A. Zinchenko Joint Institute for Nuclear Research, Dubna, Russian Federation.

I. Knowles, V. Martin, R. Sacco, A. Walker

Department of Physics and Astronomy, University of Edinburgh, Edinburgh, EH9 3JZ, U.K. ${ }^{5}$.

M. Contalbrigo, P. Dalpiaz, J. Duclos, P.L. Frabetti ${ }^{11)}$, A. Gianoli, M. Martini, L. Masetti, F. Petrucci, M. Savrié

Dipartimento di Fisica dell'Università e Sezione dell'INFN di Ferrara, I-44100 Ferrara, Italy.

A. Bizzeti ${ }^{12)}$, M. Calvetti, G. Collazuol, G. Graziani, E. Iacopini, M. Veltri ${ }^{13)}$

Dipartimento di Fisica dell'Università e Sezione dell'INFN di Firenze, I-50125 Firenze, Italy.

\footnotetext{
1) Present address: Oliver Lodge Laboratory, University of Liverpool, Liverpool L69 7ZE, U.K..

2) Present address: High Energy Accelerator Research Organization (KEK), Tsukuba, Ibaraki, 305-0801, Japan.

3) Present address: EP Division, CERN, 1211 Genève 23, Switzerland.

4) Present address: NIKHEF, PO Box 41882, 1009 DB Amsterdam, The Netherlands.

5) Funded by the U.K. Particle Physics and Astronomy Research Council.

6) On leave from Sezione dell'INFN di Firenze, I-50125 Firenze, Italy.

7) Present address: SLAC, Stanford, CA, 94309, USA.

8) On leave from Österreichische Akademie der Wissenschaften, Institut für Hochenergiephysik, A-1050 Wien, Austria.

9) Present address: University of Alberta, Edmonton Alberta T6G 2J1, Canada.

10) On leave from Joint Institute for Nuclear Research, Dubna, 141980, Russian Federation.

11) Dipartimento di Fisica e INFN Bologna, viale Berti-Pichat 6/2, I-40127 Bologna, Italy.

12) Dipartimento di Fisica dell'Università di Modena e Reggio Emilia, via G. Campi 213/A I-41100, Modena, Italy.

13) Istituto di Fisica Università di Urbino, Italy.
} 
H.G. Becker, M. Eppard, H. Fox, K. Holtz, A. Kalter, K. Kleinknecht, U. Koch, L. Köpke, P. Lopes da Silva, P. Marouelli, I. Pellmann, A. Peters, S.A. Schmidt, V. Schönharting,

Y. Schué, R. Wanke, A. Winhart, M. Wittgen Institut für Physik, Universität Mainz, D-55099 Mainz, Germany ${ }^{14)}$.

J.C. Chollet, L. Fayard, L. Iconomidou-Fayard, J. Ocariz, G. Unal, I. Wingerter-Seez

Laboratoire de l'Accélérateur Linéaire, IN2P3-CNRS, Université de Paris-Sud, 91898 Orsay, France $^{15)}$.

G. Anzivino, P. Cenci, E. Imbergamo, P. Lubrano, A. Mestvirishvili, A. Nappi, M. Pepe, M. Piccini

Dipartimento di Fisica dell'Università e Sezione dell'INFN di Perugia, I-06100 Perugia, Italy.

R. Casali, C. Cerri, M. Cirilli16) , F. Costantini, R. Fantechi, S. Giudici, I. Mannelli, G. Pierazzini, M. Sozzi

Dipartimento di Fisica, Scuola Normale Superiore e Sezione dell'INFN di Pisa, I-56100 Pisa, Italy.

J.B. Chèze, J. Cogan, M. De Beer, P. Debu, A. Formica, R. Granier de Cassagnac, E. Mazzucato, B. Peyaud, R. Turlay, B. Vallage DSM/DAPNIA - CEA Saclay, F-91191 Gif-sur-Yvette, France.

M. Holder, A. Maier, M. Ziolkowski

Fachbereich Physik, Universität Siegen, D-57068 Siegen, Germany ${ }^{17)}$.

R. Arcidiacono, C. Biino, N. Cartiglia, M. Clemencic, F. Marchetto, E. Menichetti Dipartimento di Fisica Sperimentale dell'Università e Sezione dell'INFN di Torino, I-10125 Torino, Italy.

J. Nassalski, E. Rondio, M. Szleper, W. Wislicki, S. Wronka

Soltan Institute for Nuclear Studies, Laboratory for High Energy Physics, PL-00-681 Warsaw, Poland ${ }^{18)}$.

G. Fischer, M. Jeitler, M. Markytan, G. Neuhofer, M. Pernicka, A. Taurok, L. Widhalm Österreichische Akademie der Wissenschaften, Institut für Hochenergiephysik, A-1050 Wien, Austria ${ }^{19)}$.

(Submitted to The European Physical Journal C)

14) Funded by the German Federal Minister for Research and Technology (BMBF) under contract 7MZ18P(4)TP2.

15) Funded by Institut National de Physique des Particules et de Physique Nucléaire (IN2P3), France.

16) Present address: Dipartimento di Fisica dell'Università di Roma "La Sapienza" e Sezione INFN di Roma, I-00185 Roma, Italy.

17) Funded by the German Federal Minister for Research and Technology (BMBF) under contract 056SI74.

18) Supported by the Committee for Scientific Research grants 5P03B10120, 2P03B11719 and SPUBM/CERN/P03/DZ210/2000 and using computing resources of the Interdisciplinary Center for Mathematical and Computational Modelling of the University of Warsaw.

19) Funded by the Austrian Ministry of Education, Science and Culture under contract GZ 616.360/2-IV GZ 616.363/2-VIII, and by the Fund for Promotion of Scientific Research in Austria (FWF) under contract P08929PHY. 


\begin{abstract}
The $K_{\mathrm{L}} \rightarrow \pi^{+} \pi^{-} e^{+} e^{-}$and $K_{\mathrm{S}} \rightarrow \pi^{+} \pi^{-} e^{+} e^{-}$decay modes have been studied in detail using the NA48 detector at the CERN SPS. Based on the data collected during the 1998 and 1999 run periods, a sample of $1162 K_{\mathrm{L}} \rightarrow \pi^{+} \pi^{-} e^{+} e^{-}$candidates has been observed with an expected background level of 36.9 events, yielding the branching ratio measurement $\operatorname{BR}\left(K_{\mathrm{L}} \rightarrow \pi^{+} \pi^{-} e^{+} e^{-}\right)=(3.08 \pm 0.20) \times 10^{-7}$. The distribution of events in the $\sin \phi \cos \phi$ variable, where $\phi$ is the angle between the $\pi^{+} \pi^{-}$and the $e^{+} e^{-}$ decay planes in the kaon centre of mass, is found to exhibit a large CP-violating asymmetry with the value $\mathcal{A}_{\phi}=(14.2 \pm 3.6) \%$. For the $K_{\mathrm{S}} \rightarrow \pi^{+} \pi^{-} e^{+} e^{-}$decay channel, 621 candidates have been identified in the 1999 data sample with an estimated background contribution of 0.7 event. The corresponding branching ratio has been determined to be $\mathrm{BR}\left(K_{\mathrm{S}} \rightarrow \pi^{+} \pi^{-} e^{+} e^{-}\right)=(4.71 \pm 0.32) \times 10^{-5}$. The combined value of this measurement with the published 1998 result is $\operatorname{BR}\left(K_{\mathrm{S}} \rightarrow \pi^{+} \pi^{-} e^{+} e^{-}\right)=(4.69 \pm 0.30) \times 10^{-5}$. No asymmetry is observed in this decay mode. Our results are in good agreement with theoretical predictions based on a phenomenological description of radiative kaon decays. The form factor parameters $\mathrm{a}_{1} / \mathrm{a}_{2}$ and $\tilde{\mathrm{g}}_{\mathrm{M} 1}$ in the $K_{\mathrm{L}} \rightarrow \pi^{+} \pi^{-} e^{+} e^{-}$direct emission process as well as the value of the $K^{0}$ charge radius have been extracted from the data.
\end{abstract}


Introduction

It was recognized long ago that radiative decays of neutral kaons provide an interesting ground for the study of CP non-invariance [1, 2, 3, 4]. In 1967, Dolgov and Ponomarev [4] pointed out that in the case of the $K^{0} \rightarrow \pi^{+} \pi^{-} \gamma^{*} \rightarrow \pi^{+} \pi^{-} e^{+} e^{-}$decay, large effects due to $\mathrm{CP}$ violation could be observed in the polarization of the photon. First attempts to compute the matrix element of this decay led to crude estimates of the $K_{\mathrm{L}, \mathrm{S}} \rightarrow \pi^{+} \pi^{-} e^{+} e^{-}$partial decay widths [5, 6]. More recently, Sehgal and Wanninger [7] and Heiliger and Sehgal [8] performed a detailed analysis of the $K_{\mathrm{L}} \rightarrow \pi^{+} \pi^{-} e^{+} e^{-}$differential decay rate. They showed that the angular correlation of the $e^{+} e^{-}$and $\pi^{+} \pi^{-}$planes contains an explicit CP-violating term which is sensitive to the interference between amplitudes of opposite CP.

The $K_{\mathrm{L}} \rightarrow \pi^{+} \pi^{-} e^{+} e^{-}$decay amplitude is dominated by two competing $K_{\mathrm{L}} \rightarrow \pi^{+} \pi^{-} \gamma^{*}$ components: one from the CP-violating bremsstrahlung process in which the $K_{\mathrm{L}}$ decays into $\pi^{+} \pi^{-}$where one of the pions radiates a virtual photon, the other from the CP-conserving direct emission process associated with a magnetic dipole transition. The interference of the $\mathrm{CP}$ even and CP-odd amplitudes produces a CP-violating circular polarization of the virtual photon which gives rise to an asymmetry in the distribution of the angle $\phi$ between the $e^{+} e^{-}$and the $\pi^{+} \pi^{-}$planes, in the kaon centre-of-mass system:

$$
\mathcal{A}_{\phi}=\frac{\int_{0}^{\pi / 2} \frac{\mathrm{d} \Gamma}{\mathrm{d} \phi} \mathrm{d} \phi-\int_{\pi / 2}^{\pi} \frac{\mathrm{d} \Gamma}{\mathrm{d} \phi} \mathrm{d} \phi}{\int_{0}^{\pi / 2} \frac{\mathrm{d} \Gamma}{\mathrm{d} \phi} \mathrm{d} \phi+\int_{\pi / 2}^{\pi} \frac{\mathrm{d} \Gamma}{\mathrm{d} \phi} \mathrm{d} \phi}
$$

where $\frac{\mathrm{d} \Gamma}{\mathrm{d} \phi}$ is the $K_{\mathrm{L}} \rightarrow \pi^{+} \pi^{-} e^{+} e^{-}$differential decay rate, which can be written in the general form

$$
\frac{\mathrm{d} \Gamma}{\mathrm{d} \phi}=\Gamma_{1} \cos ^{2} \phi+\Gamma_{2} \sin ^{2} \phi+\Gamma_{3} \sin \phi \cos \phi
$$

The coefficient $\Gamma_{3}$ of the asymmetric term $\sin \phi \cos \phi$ in Eq. 2 contains the contribution from interferences between amplitudes of opposite CP values. A non-zero value of $\Gamma_{3}$ constitutes therefore an unambiguous signature of $\mathrm{CP}$ violation that can be reached through the measurement of $\mathcal{A}_{\phi}$. This asymmetry, which originates mostly from $K^{0}-\overline{K^{0}}$ mixing, is predicted to be as large as $14 \%$ in $K_{\mathrm{L}} \rightarrow \pi^{+} \pi^{-} e^{+} e^{-}$decays $[7,8]$. The contribution to $\mathcal{A}_{\phi}$ from CP violation in the short-distance Z-penguin and W-box diagrams was also considered by several authors $[8,9,10]$, but was found to be negligible. Present estimates of the $K_{\mathrm{L}} \rightarrow \pi^{+} \pi^{-} e^{+} e^{-}$ branching ratio are approximately $3 \times 10^{-7}[7,8]$.

In the case of the short-lived neutral kaon, the decay amplitude is largely dominated by the $\mathrm{CP}$-even inner bremsstrahlung component. Thus, no significant asymmetry in the $\phi$ distribution is expected in the $K_{\mathrm{S}} \rightarrow \pi^{+} \pi^{-} e^{+} e^{-}$decay. The branching ratio for this decay mode can be related to the one of the $K_{\mathrm{L}} \rightarrow \pi^{+} \pi^{-} e^{+} e^{-}$inner bremsstrahlung contribution [7,8] and is predicted to be about two orders of magnitude larger than the $K_{\mathrm{L}} \rightarrow \pi^{+} \pi^{-} e^{+} e^{-}$branching ratio.

Only recently, with the advent of intense neutral kaon beams and of high-rate capability trigger and data acquisition systems, has the study of $K_{\mathrm{L}, \mathrm{S}} \rightarrow \pi^{+} \pi^{-} e^{+} e^{-}$decays become accessible to experiment. The first measurement of the $K_{\mathrm{L}} \rightarrow \pi^{+} \pi^{-} e^{+} e^{-}$branching ratio was reported in 1998 by the KTeV E799 collaboration [11] which obtained the value $\operatorname{BR}\left(K_{\mathrm{L}} \rightarrow \pi^{+} \pi^{-} e^{+} e^{-}\right)=\left(3.2 \pm 0.6_{\text {stat }} \pm 0.4_{\text {syst }}\right) \times 10^{-7}$. This result is based on a sample of 46 candidates with a background level of 9.4 events, representing $2 \%$ of the data collected in 1997. The subsequent analysis of the entire KTeV E799 data led to the observa- 
tion of $1811 K_{\mathrm{L}} \rightarrow \pi^{+} \pi^{-} e^{+} e^{-}$events and to the measurement of a CP-violating asymmetry of $\left(13.6 \pm 2.5_{\text {stat }} \pm 1.2_{\text {syst }}\right) \%$ in the $\sin \phi \cos \phi$ variable [12]. Takeuchi et al. [13] published the value $\operatorname{BR}\left(K_{\mathrm{L}} \rightarrow \pi^{+} \pi^{-} e^{+} e^{-}\right)=\left(4.4 \pm 1.3_{\text {stat }} \pm 0.5_{\text {syst }}\right) \times 10^{-7}$ from a sample of $13.5 \pm 4.0$ signal events observed at KEK. More recently, the NA48 collaboration at CERN [14] reported the first observation of the $K_{\mathrm{S}} \rightarrow \pi^{+} \pi^{-} e^{+} e^{-}$mode and measured the value $\operatorname{BR}\left(K_{\mathrm{S}} \rightarrow \pi^{+} \pi^{-} e^{+} e^{-}\right)=\left(4.5 \pm 0.7_{\text {stat }} \pm 0.4_{\text {syst }}\right) \times 10^{-5}$, using a sample of 56 events.

The possibility to probe $\mathrm{CP}$ violation effects through the polarization of the photon in the $K^{0} \rightarrow \pi^{+} \pi^{-} \gamma^{*}$ process as well as the recent experimental progress achieved in this field have aroused considerable theoretical interest $[9,10,15,16,17,18,19,20,21]$ and stimulated several calculations based on chiral perturbation theory $[22,23]$. We present in this paper the results from a study of the $K_{\mathrm{L}} \rightarrow \pi^{+} \pi^{-} e^{+} e^{-}$and $K_{\mathrm{S}} \rightarrow \pi^{+} \pi^{-} e^{+} e^{-}$decay modes performed by the NA48 experiment at the CERN SPS. Measurements of the branching ratio and asymmetry $\mathcal{A}_{\phi}$ for both channels have been obtained with data samples collected in 1998 and 1999, concurrently with the $\varepsilon^{\prime} / \varepsilon$ run. A special 2-day test dedicated in 1999 to the investigation of rare decays with an intense $K_{\mathrm{S}}$ beam allowed the statistics on the $K_{\mathrm{S}} \rightarrow \pi^{+} \pi^{-} e^{+} e^{-}$mode to be increased significantly.

\section{The NA48 neutral kaon beams}

The NA48 experiment uses a $450 \mathrm{GeV} / c$ proton beam from the CERN Super Proton Synchroton which delivers $2.38 \mathrm{~s}$ long spills every $14.4 \mathrm{~s}$ [24]. The long- and short-lived neutral kaon beams are produced from two different targets, located respectively $126 \mathrm{~m}$ and $6 \mathrm{~m}$ upstream of the beginning of the decay region. The simultaneous $K_{\mathrm{L}}$ and $K_{\mathrm{S}}$ beams used for the $\varepsilon^{\prime} / \varepsilon$ programme as well as the high-intensity $K_{\mathrm{S}}$ beam set-up are briefly described below.

\subsection{The simultaneous $K_{\mathrm{L}}$ and $K_{\mathrm{S}}$ beams}

The primary proton beam, with a nominal flux of $1.5 \times 10^{12}$ particles per spill, impinges on a $40 \mathrm{~cm}$ long, $2 \mathrm{~mm}$ diameter beryllium target, at a downward angle of $2.4 \mathrm{mrad}$ to produce the $K_{\mathrm{L}}$ beam. The charged component of the outgoing particles produced at the target is swept away by bending magnets. The neutral beam, composed mainly of photons, neutrons and longlived neutral kaons, is defined by a set of defining and cleaning collimators. The exit face of the last collimator is located $126 \mathrm{~m}$ downstream of the target, at the entrance of the fiducial kaon decay region. The primary protons which have not interacted in the target are deflected towards a bent silicon crystal [25]. A small fraction of these protons is channeled by the crystal and deflected back onto the $K_{\mathrm{L}}$ beam line. The resulting low intensity proton beam $\left(\approx 3 \times 10^{7} /\right.$ spill $)$ is then transported towards a second beryllium target, similar to the first, for the production of the $K_{\mathrm{S}}$ beam. The protons directed to the $K_{\mathrm{S}}$ target are detected by a tagging station which is used to identify the origin, $K_{\mathrm{S}}$ or $K_{\mathrm{L}}$, of the final decay products.

The $K_{\mathrm{S}}$ target is positioned $7.2 \mathrm{~cm}$ above the $K_{\mathrm{L}}$ beam axis and $120 \mathrm{~m}$ downstream of the $K_{\mathrm{L}}$ target. The $K_{\mathrm{S}}$ collimator selects secondary neutral particles at a $4.2 \mathrm{mrad}$ production angle with a divergence of $\pm 375 \mu \mathrm{rad}$. Its exit face coincides longitudinally with the one of the $K_{\mathrm{L}}$ final collimator. The $K_{\mathrm{S}}$ beam enters the fiducial decay volume $6.84 \mathrm{~cm}$ above the $K_{\mathrm{L}}$ beam. The beginning of the decay region is precisely defined on the $K_{\mathrm{S}}$ beam line by an anti-counter, called AKS, which detects all $K_{\mathrm{S}}$ decays occuring further upstream. The axes of the two beams cross at the position of the electromagnetic calorimeter with a convergence angle of $0.6 \mathrm{mrad}$.

The total flux per spill of $K_{\mathrm{L}}$ in the $K_{\mathrm{L}}$ beam entering the fiducial region is about $2 \times 10^{7}$ and the one of $K_{\mathrm{S}}$ in the $K_{\mathrm{S}}$ beam about $2 \times 10^{2}$. Fig. 1 shows a schematic view of the NA48 beam layout. 


\subsection{The high intensity $K_{\mathrm{S}}$ beam}

High intensity beams of short-lived neutral kaons can be obtained by sending the $450 \mathrm{GeV} / \mathrm{c}$ primary protons directly to the $K_{\mathrm{S}}$ target. In this configuration, the $K_{\mathrm{L}}$ target is removed whereas the bent crystal together with the tagging station are by-passed. In addition, the proton beam is attenuated and collimated to the desired intensity, far upstream of the $K_{\mathrm{S}}$ target. During a 2-day run in 1999 , the intensity of the proton beam hitting the $K_{\mathrm{S}}$ target was set to $6 \times 10^{9} /$ spill yielding a $K_{\mathrm{S}}$ flux about 200 times larger than the one obtained with the standard beam setup used for the direct $\mathrm{CP}$ violation measurement. The instantaneous rates in the various NA48 detector elements were nevertheless similar to the ones measured in the $\varepsilon^{\prime} / \varepsilon$ two-beam configuration.

\section{The NA48 detector}

The NA48 detector has been designed to measure the direct $\mathrm{CP}$ violation parameter $\operatorname{Re}\left(\varepsilon^{\prime} / \varepsilon\right)$ in $K^{0} \rightarrow 2 \pi$ decays with high precision [26, 27]. It is located downstream of the kaon decay volume which lies inside a large, $90 \mathrm{~m}$ long, vacuum tank terminated by a $0.3 \%$ radiation lengths thick Kevlar window. Starting at the centre of the Kevlar window, a $16 \mathrm{~cm}$ diameter vacuum beam pipe traverses all the detector elements to let the neutral beam pass through vacuum. The layout of the main detector is shown in Fig. 2.

\subsection{The magnetic spectrometer}

The detection of charged particles in $K_{\mathrm{L}, \mathrm{S}} \rightarrow \pi^{+} \pi^{-} e^{+} e^{-}$decays is performed using a high resolution magnetic spectrometer which consists of a dipole magnet with a horizontal transverse momentum kick of $265 \mathrm{MeV} / c$ and a set of four drift chambers [28]. Two of them are located upstream of the magnet for the measurement of the decay vertex position whereas the other two, located downstream of the magnet, are used for the bending angle determination of the tracks. The magnetic spectrometer is contained inside a tank filled with helium in order to reduce multiple scattering. Each chamber contains 8 planes of sense wires oriented in four different directions $0^{\circ}\left(\mathrm{X}, \mathrm{X}^{\prime}\right), 90^{\circ}\left(\mathrm{Y}, \mathrm{Y}^{\prime}\right),-45^{\circ}\left(\mathrm{U}, \mathrm{U}^{\prime}\right)$ and $+45^{\circ}\left(\mathrm{V}, \mathrm{V}^{\prime}\right)$, orthogonal to the beam axis. In the drift chamber located just downstream of the spectrometer magnet, only horizontal and vertical wire planes are instrumented. The momentum determination of a track is achieved with a resolution given by

$$
\frac{\sigma_{\mathrm{p}}}{\mathrm{p}}(\%)=0.48 \oplus 0.009 \mathrm{p} \quad(\mathrm{p} \text { in } \mathrm{GeV} / c) .
$$

The space resolution in each transverse coordinate is $90 \mu \mathrm{m}$ and the average efficiency per plane is better than $99 \%$.

\subsection{The scintillator hodoscope}

The precise time reference of tracks is provided by a scintillator hodoscope located downstream of the helium tank. It is composed of two planes segmented in horizontal and vertical slabs and arranged in four quadrants. The time resolution achieved is about 200 ps per track. Each quadrant also provides signals which are combined in a fast logic to be used in the first level of the trigger for charged events.

\subsection{The electromagnetic calorimeter}

The $e / \pi$ identification is obtained by comparing the momentum $\mathrm{p}$ of a track measured by the magnetic spectrometer with the energy E deposited in a quasi-homogeneous liquid krypton (LKr) calorimeter [29]. This detector has a $127 \mathrm{~cm}$ long projective tower structure which is made of copper-beryllium ribbons extending between the front and the back of the calorimeter 
with a \pm 48 mrad accordion geometry. The 13212 readout cells each have a cross-section of $2 \times 2$ $\mathrm{cm}^{2}$ at the back of the active region. The initial value of the current induced on the electrodes by the drift of the ionisation is measured using $80 \mathrm{~ns}$ FWHM pulse shapers digitized with $40 \mathrm{MHz}$ FADCs. The energy resolution of the calorimeter is

$$
\frac{\sigma_{\mathrm{E}}}{\mathrm{E}}(\%)=\frac{3.2}{\sqrt{\mathrm{E}}} \oplus \frac{9.0}{\mathrm{E}} \oplus 0.42 \quad(\mathrm{E} \text { in } \mathrm{GeV}) .
$$

The time and space resolutions achieved for $20 \mathrm{GeV}$ photons are better than $300 \mathrm{ps}$ and $1.3 \mathrm{~mm}$, respectively.

\subsection{The hadronic calorimeter and muon counters}

Behind the LKr electromagnetic calorimeter, a 6.7 nuclear interaction lengths thick calorimeter made of iron and scintillator is used to provide the energy of hadrons for the trigger. It is followed by a set of three planes of $25 \mathrm{~cm}$ wide scintillation counters shielded by $80 \mathrm{~cm}$ thick iron walls for the identification of muons.

\subsection{The AKS and AKL veto counters}

The $K_{\mathrm{S}}$ anti-counter or AKS is composed of a converter consisting of a $3 \mathrm{~mm}$ thick iridium crystal, followed by three scintillation counters. It is located at the exit of the $K_{\mathrm{S}}$ collimator to veto all upstream decays from the short-lived beam, thus providing the precise definition of the beginning of the $K_{\mathrm{S}}$ decay region. The time resolution obtained with the AKS is about $160 \mathrm{ps}$ and the inefficiency for detecting charged particles is about $2 \times 10^{-3}$. During the high intensity $K_{\mathrm{S}}$ test run, the converter was removed to minimize particle interactions in the AKS material. The $K_{\mathrm{S}}$ anti-counter was still very efficient in rejecting charged particles from upstream decays or interactions in the final collimator.

The fiducial region of the NA48 experiment is surrounded by an ensemble of seven annular iron-plastic scintillator veto counters, called AKL, to identify photons escaping the acceptance of the main detector. The time resolution of these counters is about $400 \mathrm{ps}$.

\subsection{The proton tagging station}

The proton tagging detector, located after the silicon crystal [30, 31], is made of two arrays of thin scintillation counters. This device is used to tag $K_{\mathrm{S}}$ decays by measuring very accurately the time difference between a proton in the tagging detector and an event in the main detector. Both times are reconstructed relative to a common clock running at $40 \mathrm{MHz}$. The signals of the tagging detector are digitized by $960 \mathrm{MHz}$ FADCs. The proton time resolution obtained at a proton rate of $28 \mathrm{MHz}$ is about $120 \mathrm{ps}$ and the double-pulse separation is $4 \mathrm{~ns}$.

\section{The 4-track trigger}

In order to select events compatible with decays into four charged particles, a specific algorithm was implemented in the Level 2 charged trigger (L2C), concurrently with the $\varepsilon^{\prime} / \varepsilon$ trigger [32,33]. The L2C trigger performs a fast tracking of charged particles in the spectrometer. It receives signals from the earlier Level 1 (L1) trigger stage which requires a minimum number of hits in the most upstream drift chamber and in the scintillator hodoscope, compatible with at least two tracks, together with a total energy seen in the electromagnetic and hadronic calorimeters greater than $35 \mathrm{GeV}$. The output rate of the L1 stage during the 1998 and 1999 run periods varied between 100 and $120 \mathrm{kHz}$. The measured efficiency of the L1 charged trigger for 4-track events of energy greater than $40 \mathrm{GeV}$ is $99.4 \pm 0.1 \%$. 
The L2C trigger used $200 \mathrm{MHz}$ processors in 1998 and was upgraded with $300 \mathrm{MHz}$ ones in 1999, allowing complex events to be treated more efficiently. The 4-track trigger requires at least three reconstructed space-points in each of the drift chambers 1,2 and 4 and at least two compatible 2-track vertices within a given distance along the longitudinal kaon direction. The latter requirement was set to $9 \mathrm{~m}$ in 1998 but was reduced to $3 \mathrm{~m}$ in 1999 without significant loss of efficiency. No condition on the invariant mass of the selected 4-track candidates was imposed. Depending on the run conditions, the output rate of the $\mathrm{L} 2 \mathrm{C}$ trigger varied in the 0.5$1 \mathrm{kHz}$ range, representing $5 \%$ to $10 \%$ of the total Level 2 rate of the experiment. The dead time of the L2C trigger logic was $2.9 \%$ in 1998 and $0.7 \%$ in 1999.

To determine the efficiency of the 4-track trigger, downscaled events that passed the L1 condition were recorded with a control trigger. The trigger inefficiency from the L2C algorithm itself, measured with the abundant sample of $K_{\mathrm{L}} \rightarrow \pi^{+} \pi^{-} \pi_{\mathrm{D}}^{0} \rightarrow \pi^{+} \pi^{-} e^{+} e^{-} \gamma$ events, was found to be $2.8 \%$ in 1998 and $1.9 \%$ in 1999 . The main contribution to this inefficiency is attributed to drift chamber wire inefficiencies.

Another source of inefficiency of the L2C trigger is due to the maximum latency of $102.4 \mu$ s allocated to process the events. In 1998, $27 \%$ of good 4-track events were affected by this limitation. To study possible biases introduced by these event losses, about $25 \%$ of charged triggers which exceeded the available processing time were recorded. In 1999, the fraction of events exceeding the allocated processing time was reduced to less than $2 \%$ owing to the use of faster processors.

An additional contribution of $8-12 \%$ to the trigger inefficiency comes from highmultiplicity events in the spectrometer which are produced to a large extent by accidental electromagnetic showers generated upstream of the drift chambers. Such events generate an overflow condition which resets the front-end readout buffers when more than 7 hits per plane are present within a $100 \mathrm{~ns}$ time interval [34]. The fraction of accidental high-multiplicity events, measured with pseudo-random triggers collected proportionally to beam intensity, was found to be in the $21-25 \%$ range for the $1998+1999 \varepsilon^{\prime} / \varepsilon$ run conditions, and about $30 \%$ during the high intensity $K_{\mathrm{S}}$ run.

The efficiency of the 4-track trigger, for events recorded in the presence of high multiplicities in the drift chambers was typically $65 \%$, due to missing information in the read-out buffers. The overall 4-track trigger efficiency, taking into account the above effects, was $65 \%$ in 1998 and $88 \%$ in 1999.

\section{$5 \quad$ Data samples}

A large part of the data used for the study of the $K_{\mathrm{L}, \mathrm{S}} \rightarrow \pi^{+} \pi^{-} e^{+} e^{-}$decay modes was collected during the 1998 and 1999 SPS running periods devoted to the measurement of $\operatorname{Re}\left(\varepsilon^{\prime} / \varepsilon\right)$. The use of simultaneous $K_{\mathrm{L}}$ and $K_{\mathrm{S}}$ beams allowed both $K_{\mathrm{L}} \rightarrow \pi^{+} \pi^{-} e^{+} e^{-}$and $K_{\mathrm{S}} \rightarrow \pi^{+} \pi^{-} e^{+} e^{-}$samples to be recorded concurrently. The number of 4-track triggers collected was $2.2 \times 10^{8}$ in 1998 and $3.8 \times 10^{8}$ in 1999 . The short test run performed in 1999 with a high intensity $K_{\mathrm{S}}$ beam provided an additional factor of more than 3 in the number of available $K_{\mathrm{S}}$ decays, corresponding to about $2.7 \times 10^{7}$ recorded 4-track triggers during that short period.

The $K_{\mathrm{L}} \rightarrow \pi^{+} \pi^{-} e^{+} e^{-}$and $K_{\mathrm{S}} \rightarrow \pi^{+} \pi^{-} e^{+} e^{-}$branching ratios are determined relative to that of the well-known $K_{\mathrm{L}} \rightarrow \pi^{+} \pi^{-} \pi^{0}$ mode, followed by the Dalitz decay of the neutral pion $\left(\pi_{\mathrm{D}}^{0} \rightarrow e^{+} e^{-} \gamma\right)$. This choice for the normalization has the advantage that inefficiencies in the trigger or in the event reconstruction largely cancel in the ratio.

The analysis of the $K_{\mathrm{L}, \mathrm{S}} \rightarrow \pi^{+} \pi^{-} e^{+} e^{-}$decay modes is essentially based on samples of 4-track triggers. Control or random triggers are however used to determine the trigger inefficiencies, the kaon energy spectra and to study accidental effects. In order to minimize as 
much as possible biases in the trigger or in the offline reconstruction, events having a readout overflow condition in a $\pm 312.5 \mathrm{~ns}$ time window around the event time are discarded. No effect on the measurement of the branching ratio is expected due to this rejection criterion if the accidental rate of high-multiplicity events in the drift chambers is the same for signal and normalization events. This condition has been verified to better than $0.5 \%$ by simulating the overflow appearance mechanism in the presence of accidental activity.

In the analysis discussed below, data from both 1998 and 1999 runs have been used for the study of the $K_{\mathrm{L}} \rightarrow \pi^{+} \pi^{-} e^{+} e^{-}$decays. As far as the $K_{\mathrm{S}} \rightarrow \pi^{+} \pi^{-} e^{+} e^{-}$channel is concerned, more than $90 \%$ of the total statistics originates from data recorded during the 1999 runs. The results obtained from the 1998 data have been published elsewhere [14]; they are combined with the ones of 1999, presented in this paper.

\section{Event selection and background rejection}

The selection of $K_{\mathrm{L}, \mathrm{S}} \rightarrow \pi^{+} \pi^{-} e^{+} e^{-}$and $K_{\mathrm{L}} \rightarrow \pi^{+} \pi^{-} \pi_{\mathrm{D}}^{0}$ decays relies mostly on the tracking performed in the magnetic spectrometer and also on the information obtained from the LKr calorimeter for the $e / \pi$ separation and $\pi_{\mathrm{D}}^{0}$ reconstruction.

The offline selection of both signal and normalization events requires a set of four tracks fully contained in the spectrometer and forming a vertex located in the fiducial kaon-decay region, defined along the beam direction between the AKS position $(z=0)$ and $z=84 \mathrm{~m}$. The computation of the track and vertex parameters from the hits measured in the drift chambers is based on the linear Kalman filter method [35]. The reconstruction takes into account the measured field map of the spectrometer magnet as well as multiple scattering in the Kevlar window, in the drift chambers and in helium. In addition, small track deviations due to a residual magnetic field $\left(\int \mathrm{Bdl}=20 \mathrm{G} \cdot \mathrm{m}\right)$ in the kaon decay volume, are taken into account in the reconstruction program to improve the accuracy of the measured kaon decay parameters.

Electromagnetic showers in the LKr calorimeter are found by using the digitized pulses from individual cells and by summing the energy deposited within a radius of $11 \mathrm{~cm}$. The shower position is derived from the energy-weighted sum of $3 \times 3$ cells while the shower time is obtained from the two most energetic cells. Several corrections, obtained from electron beam scans or $e^{ \pm}$ tracks from $K_{\mathrm{e} 3}$ decays, are applied at the reconstruction level to take into account second order effects: dependence of the response on the impact point inside a cell, calorimeter projectivity, overlapping showers, space charge, energy loss in the material in front of the calorimeter and residual energy non-linearity.

The following subsections describe the criteria imposed offline for the selection of $K_{\mathrm{L}, \mathrm{S}} \rightarrow$ $\pi^{+} \pi^{-} e^{+} e^{-}$and $K_{\mathrm{L}} \rightarrow \pi^{+} \pi^{-} \pi_{\mathrm{D}}^{0}$ decays.

\subsection{Selection of $K_{\mathrm{L}, \mathrm{S}} \rightarrow \pi^{+} \pi^{-} e^{+} e^{-}$decays}

The identification of $K_{\mathrm{L}, \mathrm{S}} \rightarrow \pi^{+} \pi^{-} e^{+} e^{-}$candidates requires four tracks reconstructed inside the fiducial volume of the NA48 detector. An $11 \mathrm{~cm}$ minimum radius cut around the centre of the beam pipe at the first chamber position is imposed on particles entering the spectrometer. In addition, all four tracks are required to impinge on the electromagnetic calorimeter sufficiently far from the beam pipe and the outer edge $\left(15 \mathrm{~cm}<\mathrm{R}_{\mathrm{LKr}}<120 \mathrm{~cm}\right)$ to ensure efficient electron identification with negligible energy losses. Tracks with an impact point closer than $2 \mathrm{~cm}$ to a dead calorimeter cell are rejected. For precise timing purposes, each track is required to have at least one associated hit in the scintillator hodoscope located in front of the LKr calorimeter and to lie within $8 \mathrm{~ns}$ of the event time.

Electrons are identified by requiring $\mathrm{E} / \mathrm{p} \geq 0.85$ while tracks are designated as pions if they have $\mathrm{E} / \mathrm{p}<0.85$ and no associated hit in coincidence in the muon veto counters. In order 
to further reject pion decays occuring inside the spectrometer and to suppress fake tracks due to accidental hits in the chambers, a $\chi^{2}$ value smaller than 30 is required as a track quality cut. We require also that both the electron and pion pairs have two particles of opposite charge and that the reconstructed momentum be above $2 \mathrm{GeV} / c$ for electron candidates, and above $5 \mathrm{GeV} / c$ for pions.

A vertex made of four tracks passing the above cuts is formed if each of the six combinations of pairs of tracks has a distance of closest approach smaller than $10 \mathrm{~cm}$ and a reconstructed vertex located upstream of the Kevlar window. The rejection of fake vertices made of two overlapping decays is obtained by imposing, as a vertex quality cut, a $\chi^{2}$ value smaller than 50 . The resolutions obtained on the transverse and longitudinal positions of the vertex are typically $1.8 \mathrm{~mm}$ and $55 \mathrm{~cm}$, respectively.

To identify the origin, $K_{\mathrm{L}}$ or $K_{\mathrm{S}}$, of the decay, the momentum vector of the 4-track event is extrapolated upstream to the exit face of the final collimator, where it is required to be contained within a well-defined region around the $K_{\mathrm{L}}$ or the $K_{\mathrm{S}}$ collimator holes. The angular resolution on the direction of the reconstructed kaon momentum vector is about $35 \mu \mathrm{rad}(\mathrm{rms})$. Radius cuts of $4 \mathrm{~cm}$ and $2.5 \mathrm{~cm}$ are applied around the two nominal beam centres, at the final collimator longitudinal position, for $K_{\mathrm{L}}$ and $K_{\mathrm{S}}$ decays, respectively. Data taken during the $\varepsilon^{\prime} / \varepsilon$ run were collected with simultaneous $K_{\mathrm{L}}$ and $K_{\mathrm{S}}$ beams. In order to help removing unwanted events in the $K_{\mathrm{S}}$ sample due to decays originating from the high intensity $K_{\mathrm{L}}$ beam, a $1 \mathrm{~ns}$ consistency cut on the time difference between the event and the closest signal in the $K_{\mathrm{S}}$ tagging detector is applied. This analysis cut removes less than $1 \%$ of good events but provides an extra factor of 20 in the background suppression from the $K_{\mathrm{L}}$ beam.

To remove events from beam scattering in the collimators or in the AKS, we require the centre-of-energy of the four tracks, extrapolated to the LKr calorimeter position, to lie well inside the beam pipe. For the $K_{\mathrm{L}}$ sample, a radius cut of $5 \mathrm{~cm}$ around the beam axis is applied, whereas for the $K_{\mathrm{S}}$ sample, this requirement is extended to $8 \mathrm{~cm}$. These cuts are chosen relatively wide compared to the $K_{\mathrm{L}}$ and $K_{\mathrm{S}}$ beam spot radii of $3.6 \mathrm{~cm}$ and $4.6 \mathrm{~cm}$, respectively. Events having hits in coincidence in the AKS counter are also rejected. In the case of the data taken during the high intensity $K_{\mathrm{S}}$ run, the latter cut turns out to be very effective in removing interactions and photon conversions produced in the final collimator.

$K_{\mathrm{L}, \mathrm{S}} \rightarrow \pi^{+} \pi^{-} e^{+} e^{-}$candidates are accepted if the kaon energy is larger than $40 \mathrm{GeV}$, above the threshold value set in the trigger. In the case of $K_{\mathrm{L}}$ decays, events are required to have, in addition, a reconstructed kaon energy less than $190 \mathrm{GeV}$. This offline cut ensures that background from $K_{\mathrm{S}} \rightarrow \pi^{+} \pi^{-} e^{+} e^{-}$decays due to energetic neutral kaons produced at the $K_{\mathrm{L}}$ target is kept at a negligible level. Conversely, to reduce background contributions from $K_{\mathrm{L}} \rightarrow \pi^{+} \pi^{-} e^{+} e^{-}$decays in the $K_{\mathrm{S}}$ sample, $K_{\mathrm{S}} \rightarrow \pi^{+} \pi^{-} e^{+} e^{-}$candidates are required to have their reconstructed vertex position in the $\mathrm{z}<40 \mathrm{~m}$ range.

Background events coming from $K_{\mathrm{L}, \mathrm{S}} \rightarrow \pi^{+} \pi^{-} \gamma$ decays followed by a photon conversion in the Kevlar window or in the first drift chamber are suppressed by imposing a $2 \mathrm{~cm}$ separation between the two electron tracks in the first drift chamber. This requirement also allows the events originating from a $K_{\mathrm{L}} \rightarrow \pi^{+} \pi^{-} \pi^{0}$ decay followed by the external conversion of one of the two decay photons of the $\pi^{0}$ to be rejected.

In order to suppress background from accidental overlaps of $K_{\mathrm{L}, \mathrm{S}} \rightarrow \pi^{+} \pi^{-}$decays and photon conversions in the collimators or the detector material in front of the first chamber, events are eliminated if the reconstructed $\pi^{+} \pi^{-}$invariant mass is found to lie between 490.7 and 504.7 MeV/c $c^{2}$. Moreover, the measured time of a pion pair is required to be compatible within $1.5 \mathrm{~ns}$ with the time measured for the lepton pair. Accidental background in both $K_{\mathrm{L}}$ and $K_{\mathrm{S}}$ samples is further reduced by rejecting events with an extra track measured in the spectrometer 
within $1.5 \mathrm{~ns}$ of the event time. In the $K_{\mathrm{L}}$ beam, a residual source of accidental background comes from two quasi-simultaneous $K_{\mathrm{e} 3}$ decays with opposite pion (electron) charge. In order to reduce such a contribution, we require, for decays originating from the $K_{\mathrm{L}}$ target, the $\pi^{+} e^{-}$ and $\pi^{-} e^{+}$vertices to have their relative vertex times within a $\pm 1.5 \mathrm{~ns}$ coincidence window.

Possible background contributions from $\Xi^{0} \rightarrow \Lambda \pi_{D}^{0}$ decays can be suppressed by removing events compatible with a $\Lambda \rightarrow p \pi$ decay. Four-track candidates with the two hadrons having a $p \pi$ invariant mass within $4 \mathrm{MeV} / c^{2}$ of the $\Lambda$ mass value are eliminated.

A potentially important source of background to the $K_{\mathrm{L}, \mathrm{S}} \rightarrow \pi^{+} \pi^{-} e^{+} e^{-}$channels comes from $K_{\mathrm{L}} \rightarrow \pi^{+} \pi^{-} \pi_{\mathrm{D}}^{0}$ decays when the photon from the $\pi^{0}$ Dalitz decay escapes detection. This is particularly true in the case of the $K_{\mathrm{L}}$ mode since $K_{L} \rightarrow \pi^{+} \pi^{-} \pi_{D}^{0}$ decays occur at a rate which is almost four orders of magnitude larger than the $K_{\mathrm{L}} \rightarrow \pi^{+} \pi^{-} e^{+} e^{-}$one. For $K_{\mathrm{S}}$ decays, the situation is more favourable owing to the larger branching ratio of the $K_{\mathrm{S}} \rightarrow \pi^{+} \pi^{-} e^{+} e^{-}$ channel and the smaller decay length for the long-lived neutral kaons produced at the $K_{\mathrm{S}}$ target station.

\subsection{1 $K_{\mathrm{L}} \rightarrow \pi^{+} \pi^{-} e^{+} e^{-}$}

In the $K_{\mathrm{L}}$ sample, a large fraction of events with a missing particle are suppressed by requiring that the square of the total transverse momentum $\mathrm{p}_{\perp}^{2}$ of the observed decay products relative to the line of flight joining the centre of the $K_{\mathrm{L}}$ target to the parent kaon decay point be less than $5 \times 10^{-4} \mathrm{GeV}^{2} / c^{2}$. The resolution obtained on $\mathrm{p}_{\perp}^{2}$ is about $9 \times 10^{-5} \mathrm{GeV}^{2} / c^{2}$ for $K_{\mathrm{L}} \rightarrow \pi^{+} \pi^{-} e^{+} e^{-}$decays. This condition, when applied after the centre of gravity cut, removes about $70 \%$ of the remaining $K_{\mathrm{L}} \rightarrow \pi^{+} \pi^{-} \pi_{\mathrm{D}}^{0}$ decays, while more than $98 \%$ of good events are kept. Moreover, events associated with a hit in one of the scintillator pockets (AKL) around the detector are rejected.

In order to further suppress events from $K_{\mathrm{L}} \rightarrow \pi^{+} \pi^{-} \pi_{\mathrm{D}}^{0}$ decays, the well-known kinematic variable $\mathrm{p}_{0}^{\prime 2}[36]$ is used in the offline analysis:

$$
\begin{gathered}
\mathrm{p}_{0}^{\prime 2}=\frac{1}{4\left(\mathrm{M}_{\pi \pi}^{2}+\left(\mathrm{p}_{\perp}^{2}\right)_{\pi \pi}\right)}\left\{\left(\mathrm{M}_{K}^{2}-\mathrm{M}_{\pi^{0}}^{2}-\mathrm{M}_{\pi \pi}^{2}\right)^{2}\right. \\
\left.-4 \mathrm{M}_{\pi^{0}}^{2} \mathrm{M}_{\pi \pi}^{2}-4\left(\mathrm{p}_{\perp}^{2}\right)_{\pi \pi} \mathrm{M}_{K}^{2}\right\} .
\end{gathered}
$$

In the equation above, $\mathrm{M}_{\pi \pi}$ and $\left(\mathrm{p}_{\perp}\right)_{\pi \pi}$ are, respectively, the invariant mass and the transverse momentum of the $\pi^{+} \pi^{-}$pair, $\mathrm{M}_{K}=497.7 \mathrm{MeV} / c^{2}$ is the kaon mass and $\mathrm{M}_{\pi^{0}}=135.0 \mathrm{MeV} / c^{2}$ the neutral pion mass. In the $K_{\mathrm{L}} \rightarrow \pi^{+} \pi^{-} \pi^{0}$ hypothesis, the $\mathrm{p}_{0}^{\prime 2}$ variable represents the square of the longitudinal momentum of the kaon in the reference frame where the longitudinal momentum of the $\pi^{+} \pi^{-}$pair is zero. Except for resolution effects, $\mathrm{p}_{0}^{\prime 2}$ is greater than zero for $K_{\mathrm{L}} \rightarrow \pi^{+} \pi^{-} \pi_{\mathrm{D}}^{0}$ decays while it is mostly negative for $K_{\mathrm{L}} \rightarrow \pi^{+} \pi^{-} e^{+} e^{-}$events. The requirement that candidates have a $\mathrm{p}_{0}^{\prime 2}$ value below $-6 \times 10^{-3} \mathrm{GeV}^{2} / \mathrm{c}^{2}$ removes more than $99 \%$ of the remaining unwanted events but keeps about $94 \%$ of good $K_{\mathrm{L}} \rightarrow \pi^{+} \pi^{-} e^{+} e^{-}$events.

Finally, we require the invariant mass $\mathbf{M}_{\pi \pi e e}$ of the four track candidates to be compatible with the kaon mass value. In order to keep the background to signal ratio at a level of a few percent in the $K_{\mathrm{L}}$ sample, we restrict $K_{\mathrm{L}} \rightarrow \pi^{+} \pi^{-} e^{+} e^{-}$candidates to lie in the $485.7<\mathrm{M}_{\pi \pi e e}$ $<507.7 \mathrm{MeV} / c^{2}$ range. Fig. 3 shows the invariant mass $\mathrm{M}_{\pi \pi e e}$ distribution for $K_{\mathrm{L}} \rightarrow \pi^{+} \pi^{-} e^{+} e^{-}$ events after all other selection criteria have been applied. The total number of candidates in the signal region is 1162 . Their distribution, which exhibits a non-gaussian tail in the low-mass side of the peak due to radiative effects, is well reproduced by the Monte Carlo simulation. 
The distribution of events in the $\mathrm{p}_{0}^{\prime 2}$ variable is shown in Fig. 4 after all other selection cuts are applied. The steep rise close to zero is due to residual $K_{\mathrm{L}} \rightarrow \pi^{+} \pi^{-} \pi_{\mathrm{D}}^{0}$ background events. Below $\mathrm{p}_{0}^{\prime 2}=-6 \times 10^{-3} \mathrm{GeV}^{2} / c^{2}, K_{\mathrm{L}} \rightarrow \pi^{+} \pi^{-} e^{+} e^{-}$events are cleanly identified.

The contamination in the signal region from $K_{\mathrm{L}} \rightarrow \pi^{+} \pi^{-} \pi_{\mathrm{D}}^{0}$ decays has been evaluated using a Monte Carlo simulation. For this purpose, $3 \times 10^{8} K_{\mathrm{L}} \rightarrow \pi^{+} \pi^{-} \pi_{\mathrm{D}}^{0}$ decays have been generated in the fiducial decay region and all selection criteria described above applied to the reconstructed events. The number of background events in the $485.7<\mathrm{M}_{\pi \pi e e}<507.7 \mathrm{MeV} / \mathrm{c}^{2}$ region is found to be $32.9 \pm 5.0$. Overlapping $K_{\mathrm{e} 3}$ decays contribute mostly in the high mass region of the $K_{\mathrm{L}} \rightarrow \pi^{+} \pi^{-} e^{+} e^{-}$spectrum. In order to evaluate their contribution, samples of accidental 4-track events having same pion and same electron charges $\left(\pi^{+} \pi^{+} \mathrm{e}^{-} \mathrm{e}^{-}\right.$or $\left.\pi^{-} \pi^{-} \mathrm{e}^{+} \mathrm{e}^{+}\right)$ have been investigated. The contamination from double $K_{\mathrm{e} 3}$ to the signal region is estimated to be $4.0 \pm 2.0$ events. Backgrounds due to $K_{\mathrm{L}} \rightarrow \pi^{+} \pi^{-} \gamma$ radiative decays followed by photon conversion in the Kevlar window or to $K_{\mathrm{L}} \rightarrow \pi^{+} \pi^{-}$decays with an accidental photon conversion in the detector are found to be negligible. The total number of background events from the different sources described above is estimated to be $36.9 \pm 5.9$ in the signal region. The relative contributions of the two main sources, the $K_{\mathrm{L}} \rightarrow \pi^{+} \pi^{-} \pi_{\mathrm{D}}^{0}$ and the overlapping $K_{\mathrm{e} 3}$ decays, are shown in Fig. 3.

\subsection{2 $K_{\mathrm{S}} \rightarrow \pi^{+} \pi^{-} e^{+} e^{-}$}

In the $K_{\mathrm{S}}$ sample, where the background level from $K_{\mathrm{L}} \rightarrow \pi^{+} \pi^{-} \pi_{\mathrm{D}}^{0}$ decays is strongly reduced, looser offline cuts in the $\mathrm{p}_{\perp}^{2}$ and $\mathrm{p}_{0}^{\prime 2}$ variables are used. Since the distance between the production target and the measured kaon decay point is much smaller for $K_{\mathrm{S}}$ than for $K_{\mathrm{L}}$, the precision on the $\mathrm{p}_{\perp}^{2}$ determination is expected to be significantly worse. For $K_{\mathrm{S}} \rightarrow \pi^{+} \pi^{-} e^{+} e^{-}$ events, the resolution obtained on $\mathrm{p}_{\perp}^{2}$ is about $3 \times 10^{-3} \mathrm{GeV}^{2} / c^{2}$. Candidates in the $K_{\mathrm{S}}$ sample are rejected if $\mathrm{p}_{\perp}^{2}$ is greater than $2 \times 10^{-2} \mathrm{GeV}^{2} / c^{2}$ or $\mathrm{p}_{0}^{\prime 2}$ above $5 \times 10^{-3} \mathrm{GeV}^{2} / c^{2}$. Furthermore, we impose the condition $477.7<\mathrm{M}_{\pi \pi e e}<512.7 \mathrm{MeV} / c^{2}$ on the remaining candidates.

The distribution of $K_{\mathrm{S}} \rightarrow \pi^{+} \pi^{-} e^{+} e^{-}$events in the $\mathrm{p}_{0}^{\prime 2}$ vs. $\mathrm{M}_{\pi \pi e e}$ region, after having applied all other analysis cuts, is shown in Fig. 5(a) . The very good separation of $K_{\mathrm{L}} \rightarrow$ $\pi^{+} \pi^{-} \pi_{\mathrm{D}}^{0}$ decays allows the $K_{\mathrm{L}} \rightarrow \pi^{+} \pi^{-} e^{+} e^{-}$events to be clearly identified. The total number of candidates observed in the signal region is 621. Fig. 5(b) shows their invariant mass $\mathbf{M}_{\pi \pi e e}$ distribution. Background contamination in the signal region due to $K_{\mathrm{L}} \rightarrow \pi^{+} \pi^{-} \pi_{\mathrm{D}}^{0}$ events has been evaluated by Monte Carlo simulation and amounts to $0.7_{-0.7}^{+1.4}$ event.

\section{2 $\quad K_{\mathrm{L}} \rightarrow \pi^{+} \pi^{-} \pi_{\mathrm{D}}^{0}$ decays}

The selection of $K_{\mathrm{L}} \rightarrow \pi^{+} \pi^{-} \pi_{\mathrm{D}}^{0}$ events originating from the $K_{\mathrm{L}}$ or the $K_{\mathrm{S}}$ targets is very similar to the one for the $K_{\mathrm{L}, \mathrm{S}} \rightarrow \pi^{+} \pi^{-} e^{+} e^{-}$events. We require, in addition to the four identified charged particles, the presence of an isolated cluster in the $\mathrm{LKr}$ calorimeter, within $4 \mathrm{~ns}$ of the 4-track event and with an energy greater than $2 \mathrm{GeV}$, well above the detector noise of $100 \mathrm{MeV}$ per cluster. The distance of the cluster to any dead cell is required to be greater than $2 \mathrm{~cm}$ and the distance to any of the four impact points of the charged particles on the $\mathrm{LKr}$ to be greater than $15 \mathrm{~cm}$. Candidates are accepted if the reconstructed $e^{+} e^{-} \gamma$ invariant mass is in the $110-150 \mathrm{MeV} / c^{2}$ range, compatible with the $\pi^{0}$ mass value. The origin of the $K_{\mathrm{L}} \rightarrow \pi^{+} \pi^{-} \pi_{\mathrm{D}}^{0}$ is assigned in a similar way to the $K_{\mathrm{L}} \rightarrow \pi^{+} \pi^{-} e^{+} e^{-}$selection, by extrapolating the total momentum vector of the $\pi^{+} \pi^{-} e^{+} e^{-} \gamma$ state to the final collimator exit face. Moreover, depending on the origin, $K_{\mathrm{L}}$ beam or $K_{\mathrm{S}}$ beam, of the event, identical analysis cuts as the ones applied to the $K_{\mathrm{L}, \mathrm{S}} \rightarrow \pi^{+} \pi^{-} e^{+} e^{-}$samples have been imposed on the $\pi^{+} \pi^{-} e^{+} e^{-} \gamma$ final state for the centre of gravity, $\mathrm{p}_{\perp}^{2}$ and invariant mass variables. 
After all selection criteria have been applied, the total number of $K_{\mathrm{L}} \rightarrow \pi^{+} \pi^{-} \pi_{\mathrm{D}}^{0}$ events originating from the $K_{\mathrm{L}}$ target is found to be $2.83 \times 10^{6}$. Background due to the $K_{\mathrm{L}} \rightarrow \pi^{+} \pi^{-} \pi^{0}$ decays followed by a photon conversion in the Kevlar window has been estimated to be less than $0.2 \%$. The corresponding number of $K_{\mathrm{L}} \rightarrow \pi^{+} \pi^{-} \pi_{\mathrm{D}}^{0}$ candidates in the $K_{\mathrm{S}}$ beam, adding samples from both $1999 \varepsilon^{\prime} / \varepsilon$ and high intensity $K_{\mathrm{S}}$ runs, is 1403 . For the data collected with the two-beam configuration, the contamination in the $K_{\mathrm{S}}$ sample of $K_{\mathrm{L}} \rightarrow \pi^{+} \pi^{-} \pi_{\mathrm{D}}^{0}$ decays originating from the $K_{\mathrm{L}}$ target has been estimated to be $7.3 \pm 1.4$ from the study of the accidental activity in the tagging detector.

The $e^{+} e^{-} \gamma$ and $\pi^{+} \pi^{-} e^{+} e^{-} \gamma$ invariant mass distributions for identified $K_{\mathrm{L}} \rightarrow \pi^{+} \pi^{-} \pi_{\mathrm{D}}^{0}$ decays are shown in Fig. 6. The measured mass resolutions are respectively $2.0 \mathrm{MeV} / c^{2}$ and $1.5 \mathrm{MeV} / c^{2}$. Fig. 7 illustrates the clean separation obtained in the $\mathrm{Z}_{\mathrm{vtx}}-\mathrm{Y}_{\mathrm{vtx}}$ plane of the decay vertex position for $K_{\mathrm{L}, \mathrm{S}} \rightarrow \pi^{+} \pi^{-} e^{+} e^{-}$and $K_{\mathrm{L}} \rightarrow \pi^{+} \pi^{-} \pi_{\mathrm{D}}^{0}$ events produced at the $K_{\mathrm{L}}$ and $K_{\mathrm{S}}$ targets. For kaons produced at the $K_{\mathrm{S}}$ target, the beginning of the decay region is defined by the AKS veto signal. Thus, due to resolution effects, a small fraction of good events in the $K_{\mathrm{S}}$ beam are found to lie at negative values of the reconstructed longitudinal vertex position (Fig. 7(b) and (d)). For events in the $K_{\mathrm{L}}$ beam, a cut at $\mathrm{Z}_{\mathrm{vtx}}=0$ is used instead (Fig. 7(a) and (c)). The difference in the proper decay time distribution between $K_{\mathrm{L}} \rightarrow \pi^{+} \pi^{-} \pi_{\mathrm{D}}^{0}$ and $K_{\mathrm{S}} \rightarrow \pi^{+} \pi^{-} e^{+} e^{-}$ events originating from the $K_{\mathrm{S}}$ target is shown in Fig. 8.

\section{Acceptance calculation}

The acceptances for the $K_{\mathrm{L}, \mathrm{S}} \rightarrow \pi^{+} \pi^{-} e^{+} e^{-}$and $K_{\mathrm{L}} \rightarrow \pi^{+} \pi^{-} \pi_{\mathrm{D}}^{0}$ decay channels have been computed using a detailed Monte Carlo program based on GEANT [37]. Particle interactions in the detector material as well as the reponse functions of the different detector elements are taken into account in the simulation. In particular, the drift chamber wire inefficiencies measured during data taking are introduced before the event reconstruction. Shower libraries for photons, electrons and charged pions are used to describe the response of the calorimeters.

Kaon decays in the detector fiducial region are generated using production spectra at the $K_{\mathrm{L}}$ and $K_{\mathrm{S}}$ targets which have been determined from the analysis of the abundant $K_{\mathrm{L}} \rightarrow$ $\pi^{+} \pi^{-} \pi_{\mathrm{D}}^{0}$ and $K_{\mathrm{S}} \rightarrow \pi^{+} \pi^{-}$samples, respectively. The kaon momentum spectrum used for the acceptance calculation stretches between $40 \mathrm{GeV} / c$ and $190 \mathrm{GeV} / c$ for the $K_{\mathrm{L}}$ beam, while for kaons originating from the $K_{\mathrm{S}}$ target, all momenta above $40 \mathrm{GeV} / c$ are considered. We have implemented the PHOTOS code [38] in the simulation program to take into account radiative effects in the acceptance calculation for both the signal and the normalization channels. This algorithm provides the corrections from QED bremsstrahlung in the leading-logarithmic approximation with the proper soft photon behaviour taken into account. A cut-off value of $1 \mathrm{MeV}$ in the rest frame of the parent of the radiating charged particle has been used for the emitted photon. As a check of the method, we have applied the PHOTOS code to simulated $\pi^{0} \rightarrow e^{+} e^{-} \gamma$ decays and verified the consistency of the results with existing calculations of radiative corrections for this process [39].

The value of the acceptance depends on the decay matrix element of the process investigated. For the normalization channel, we have used the current experimental values of the $K_{\mathrm{L}} \rightarrow \pi^{+} \pi^{-} \pi^{0}$ decay parameters and of the $\pi^{0}$ electromagnetic form factor [40]. After all selection criteria discussed in the previous section have been applied to the reconstructed Monte Carlo events, the acceptances for $K_{\mathrm{L}} \rightarrow \pi^{+} \pi^{-} \pi_{\mathrm{D}}^{0}$ decays originating from the $K_{\mathrm{L}}$ and the $K_{\mathrm{S}}$ targets are found to be $1.80 \%$ and $1.85 \%$, respectively. The relative uncertainties on these values, due to the statistical precision of the Monte Carlo samples, are better than 4 per mille.

The generation of $K_{\mathrm{L}, \mathrm{S}} \rightarrow \pi^{+} \pi^{-} e^{+} e^{-}$events is based on the phenomenological model developed in Ref. [8]. In the case of $K_{\mathrm{S}}$, the only contribution comes from the inner bremsstrahlung 
process which can be related to the well-measured $K_{\mathrm{S}} \rightarrow \pi^{+} \pi^{-}$decay rate. The computed acceptance for the $K_{\mathrm{S}} \rightarrow \pi^{+} \pi^{-} e^{+} e^{-}$decay channel is $\left(3.51 \pm 0.01_{\text {stat }}\right) \%$. Fig. 9 shows the comparison between data and Monte Carlo of the $\mathbf{M}_{\pi \pi}$ and $\mathbf{M}_{e e}$ spectra for $K_{\mathrm{S}} \rightarrow \pi^{+} \pi^{-} e^{+} e^{-}$ decays. These distributions exhibit a shape characteristic of the inner bremsstrahlung process.

As far as the $K_{\mathrm{L}} \rightarrow \pi^{+} \pi^{-} e^{+} e^{-}$decay is concerned, the main contributions to the decay matrix element are the $\mathrm{CP}$-violating inner bremsstrahlung term and the $\mathrm{CP}$-conserving amplitude associated with the emission of an M1 photon at the $\pi^{+} \pi^{-}$decay vertex. In the model of Ref. [8] the latter contribution is described by the coupling $g_{M 1}$. It has been shown recently, however, that a form factor, similar to that used to describe the $K_{\mathrm{L}} \rightarrow \pi^{+} \pi^{-} \gamma$ decay, is required in the M1 direct emission amplitude in order to describe the measured virtual photon energy spectrum in $K_{\mathrm{L}} \rightarrow \pi^{+} \pi^{-} e^{+} e^{-}$decays [12]. This energy-dependent coupling, which takes into account the $\rho$ vector meson intermediate state, has the form:

$$
\mathcal{F}=\tilde{\mathrm{g}}_{\mathrm{M} 1}\left[1+\frac{\mathrm{a}_{1} / \mathrm{a}_{2}}{\left(\mathrm{M}_{\rho}^{2}-\mathrm{M}_{K}^{2}\right)+2 \mathrm{M}_{K} \mathrm{E}_{\gamma}^{*}}\right],
$$

where $\mathrm{M}_{\rho}=769.3 \mathrm{MeV} / c^{2}$ is the mass of the $\rho$ meson, $\mathrm{E}_{\gamma}^{*}$ is the energy of the virtual photon in the kaon centre of mass and $\tilde{g}_{\mathrm{M} 1}$ and $\mathrm{a}_{1} / \mathrm{a}_{2}$ are parameters to be determined experimentally. The $\mathrm{KTeV}$ experiment [12] has performed a detailed analysis of $K_{\mathrm{L}} \rightarrow \pi^{+} \pi^{-} e^{+} e^{-}$decays and extracted from the data the values $\mathrm{a}_{1} / \mathrm{a}_{2}=\left(-0.720 \pm 0.028_{\text {stat }} \pm 0.009_{\text {syst }}\right) \mathrm{GeV}^{2}$ and $\tilde{\mathrm{g}}_{\mathrm{M} 1}=1.35_{-0.17}^{+0.20}$ stat $\pm 0.04_{\text {syst }}$. More recently, the study of the $K_{\mathrm{L}} \rightarrow \pi^{+} \pi^{-} \gamma$ direct emission vertex by the same collaboration [41] has yielded the value $\mathrm{a}_{1} / \mathrm{a}_{2}=(-0.737 \pm 0.034) \mathrm{GeV}^{2}$, in close agreement with their previous measurement.

A third contribution to the $K_{\mathrm{L}} \rightarrow \pi^{+} \pi^{-} e^{+} e^{-}$amplitude, associated with the $K^{0}$ charge radius process, is also included in the matrix element. It is described by the parameter $\mathrm{g}_{\mathrm{P}}=$ $-\frac{1}{3}<\mathrm{R}^{2}>_{K^{0}} \mathrm{M}_{K}^{2}$ which has the value $\mathrm{g}_{\mathrm{P}}=0.15$ in Ref. [8]. The charge radius term represents, however, only a few percent of the total $K_{\mathrm{L}} \rightarrow \pi^{+} \pi^{-} e^{+} e^{-}$branching ratio. Amplitudes describing the $\mathrm{CP}$-violating $\mathrm{E} 1$ direct photon emission or direct $\mathrm{CP}$ violation have been turned off in the model as they give negligible contributions to the decay rate.

We have determined experimentally the parameters $a_{1} / a_{2}$ and $\tilde{g}_{M 1}$ involved in the form factor for the M1 direct emission process as well as the $\mathrm{g}_{\mathrm{P}}$ coupling by fitting the $K_{\mathrm{L}} \rightarrow$ $\pi^{+} \pi^{-} e^{+} e^{-}$data using the following log-likelihood function:

$$
\ln \mathcal{L}(\alpha)=\sum_{\mathrm{i}=1}^{\mathrm{N}} \ln \left[\frac{\mathrm{A}\left(\beta_{i}\right) \frac{\mathrm{d} \Gamma\left(\beta_{\mathrm{i}}, \alpha\right)}{\mathrm{d} \beta}}{\int_{\varepsilon_{\beta}} \mathrm{A}(\beta) \frac{\mathrm{d} \Gamma(\beta, \alpha)}{\mathrm{d} \beta} \mathrm{d} \beta}\right]
$$

In the equation above, $\mathrm{A}(\beta)$ is the acceptance function that depends on the five independent kinematical variables $\beta=\left\{\mathrm{M}_{\pi \pi}, \mathrm{M}_{e e}, \phi, \theta_{e^{+}}, \theta_{\pi^{+}}\right\}$, and $\frac{\mathrm{d} \Gamma(\beta, \alpha)}{\mathrm{d} \beta}$ is the differential decay rate expressed as a function of $\beta$ and the parameters $\alpha=\left\{\mathrm{a}_{1} / \mathrm{a}_{2}, \tilde{g}_{\mathrm{M} 1}, \mathrm{~g}_{\mathrm{P}}\right\}$. The other model parameters entering the computation of the decay rate are taken from Refs. [8] and [40]. As in Ref. [8], $\theta_{e^{+}}$is defined as the angle between the $e^{+}$and the $\pi^{+} \pi^{-}$direction in the $e^{+} e^{-}$centreof-mass system, and $\theta_{\pi^{+}}$, as the angle between the $\pi^{+}$and the $e^{+} e^{-}$direction in the $\pi^{+} \pi^{-}$ centre-of-mass reference. The expression inside the brackets in Eq. 7 represents, for a given event, the product of the decay probability and the acceptance, normalized over the entire phase space $\varepsilon_{\beta}$.

To reduce possible biases due to residual background or non-Gaussian tails, only $K_{\mathrm{L}} \rightarrow$ $\pi^{+} \pi^{-} e^{+} e^{-}$events having a reconstructed invariant mass $\mathrm{M}_{\pi \pi e e}$ in $\mathrm{a} \pm 4 \mathrm{MeV} / c^{2}$ interval around the $K_{\mathrm{L}}$ mass are used in the fitting procedure. This requirement removes $12.1 \%$ of the events but 
Table 1: Systematic uncertainties on the extracted $\mathrm{a}_{1} / \mathrm{a}_{2}, \tilde{\mathrm{g}}_{\mathrm{M} 1}$ and $\mathrm{g}_{\mathrm{P}}$ parameters.

\begin{tabular}{lccc}
\hline Source & $\begin{array}{c}\mathrm{a}_{1} / \mathrm{a}_{2} \\
\left(\mathrm{GeV}^{2}\right)\end{array}$ & $\tilde{\mathrm{g}}_{\mathrm{M} 1}$ & $\mathrm{~g}_{\mathrm{P}}$ \\
\hline Model parameters & 0.001 & 0.010 & 0.002 \\
MC normalization & 0.003 & 0.007 & 0.002 \\
Background & 0.013 & 0.013 & 0.007 \\
Resolution effects & 0.011 & 0.044 & 0.003 \\
Radiative corrections & 0.017 & 0.049 & 0.017 \\
\hline Total & 0.024 & 0.068 & 0.019 \\
\hline
\end{tabular}

improves the signal to background ratio by more than a factor of 3 . The search for the maximum likelihood for the considered data set has been carried out using the MINUIT code [42]. The acceptance function in Eq. 7 is provided by a sample of $4.80 \times 10^{5}$ Monte Carlo events contained in the detector acceptance and generated with the initial parameters $\alpha=\left\{-0.72 \mathrm{GeV}^{2}, 1.35,0.15\right\}$. No radiative correction has been applied to this normalization sample. During the iteration procedure, each event is properly re-weighted according to the current set of $\alpha$ values to ensure proper normalization of the likelihood function.

The maximum likelihood is found at the values $\mathrm{a}_{1} / \mathrm{a}_{2}=-0.81_{-0.13}^{+0.07} \mathrm{GeV}^{2}, \tilde{\mathrm{g}}_{\mathrm{M} 1}=$ $0.99_{-0.27}^{+0.28}$ and $\mathrm{g}_{\mathrm{P}}=0.19 \pm 0.04$, where the uncertainties are purely statistical. A high correlation coefficient of 0.979 between the $a_{1} / a_{2}$ and $\tilde{g}_{M 1}$ parameters is obtained in the determination of the form factor. Our result is consistent with the values of $a_{1} / a_{2}$ and $\tilde{g}_{M 1}$ measured by the $\mathrm{KTeV}$ experiment. The average value of the form factor for the data is $\langle\mathcal{F}\rangle=-0.78 \pm 0.05$, in agreement with the constant coupling $\left|\mathrm{g}_{\mathrm{M} 1}\right|=0.76$ used in the model of Heiliger and Sehgal [8].

Fig. 10(a) shows the measured $\mathrm{M}_{\pi \pi}$ spectrum for $K_{\mathrm{L}} \rightarrow \pi^{+} \pi^{-} e^{+} e^{-}$decays after background subtraction. It is compared to the Monte Carlo prediction obtained with the fitted parameters together with calculations using the parameters of Refs. [8] and [12]. As expected, the inclusion of an energy-dependent term in the M1 direct emission amplitude improves significantly the agreement between data and Monte Carlo. The corresponding distributions in the $\mathrm{M}_{e e}$ variable are shown in Fig. 10(b).

Different sources of systematic uncertainties that can affect the determination of the $\mathrm{a}_{1} / \mathrm{a}_{2}, \tilde{\mathrm{g}}_{\mathrm{M} 1}$ and $\mathrm{g}_{\mathrm{P}}$ parameters have been investigated. Biases due to background contamination in the event sample have been evaluated by changing the selection criteria to vary the fraction of unwanted events. Similarly, samples of simulated $K_{\mathrm{L}} \rightarrow \pi^{+} \pi^{-} e^{+} e^{-}$events with and without background contamination have been used in the fitting procedure to check the stability of the results.

Since resolution effects can also spoil the determination of the $\mathrm{a}_{1} / \mathrm{a}_{2}, \tilde{g}_{\mathrm{M} 1}$ and $\mathrm{g}_{\mathrm{P}}$ parameters, the corresponding systematic uncertainties have been evaluated by re-fitting the data sample several times, after smearing the five kinematical variables according to resolution functions determined by Monte Carlo. The sensitivity of the extracted parameters to radiative corrections has been calculated by comparing results from fits of Monte Carlo samples generated with and without the inclusion of radiative effects in the final state.

Contributions from the limited statistical precision of the Monte Carlo sample used for normalization of the log-likelihood function as well as systematic effects attributed to the uncertainty in the model input parameters, like $\mathrm{BR}\left(K_{\mathrm{S}} \rightarrow \pi^{+} \pi^{-}\right)$, the $\mathrm{CP}$ violation parameters $\left|\eta_{+-}\right|$ 
Table 2: Signal and normalization yields with the corresponding acceptances for the $K_{\mathrm{L}}$ sample.

\begin{tabular}{lcccc}
\hline Data sample & $\mathrm{N}_{\pi \pi \text { ee }}^{\mathrm{L}}$ & $\begin{array}{r}\mathrm{N}_{\pi \pi \pi_{\mathrm{D}}^{0}}^{\mathrm{L}} \\
\left(10^{6}\right)\end{array}$ & $\begin{array}{r}\mathrm{A}_{\pi \pi \mathrm{ee}}^{\mathrm{L}} \\
(\%)\end{array}$ & $\begin{array}{r}\mathrm{A}_{\pi \pi \pi_{\mathrm{D}}^{0}}^{\mathrm{L}} \\
(\%)\end{array}$ \\
\hline $1998 \varepsilon^{\prime} / \varepsilon$ & 303.4 & 0.738 & & \\
$1999 \varepsilon^{\prime} / \varepsilon$ & 821.7 & 2.088 & & 1.80 \\
\hline
\end{tabular}

Table 3: Signal and normalization yields with the corresponding acceptances for the $K_{\mathrm{S}}$ sample. The differences in the acceptance values between the 1998 and 1999 samples are mainly due to the implementation in 1999 of an $11 \mathrm{~cm}$ minimum radius cut at the first chamber position, and to the rejection in 1998 of $K_{\mathrm{L}} \rightarrow \pi^{+} \pi^{-} \pi_{\mathrm{D}}^{0}$ events having $\mathrm{Y}_{\mathrm{vtx}}<4 \mathrm{~cm}$.

\begin{tabular}{lcccc}
\hline Data sample & $\mathrm{N}_{\pi \pi \mathrm{ee}}^{\mathrm{S}}$ & $\mathrm{N}_{\pi \pi \pi_{\mathrm{D}}^{0}}^{\mathrm{S}}$ & $\begin{array}{r}\mathrm{A}_{\pi \pi \mathrm{ee}}^{\mathrm{S}} \\
(\%)\end{array}$ & $\begin{array}{r}\mathrm{A}_{\pi \pi \pi_{\mathrm{D}}^{0}}^{\mathrm{S}} \\
(\%)\end{array}$ \\
\hline $1998 \varepsilon^{\prime} / \varepsilon[14]$ & 55.7 & 103.5 & 3.70 & 1.56 \\
\hline $1999 \varepsilon^{\prime} / \varepsilon$ & 96.9 & 247.7 & & \\
$1999 \mathrm{HI} K_{\mathrm{S}}$ & 523.4 & 1148.0 & & \\
\hline
\end{tabular}

and $\phi_{+-}$, or the pion-pion phase-shifts $\delta_{0}$ and $\delta_{1}$, have also been estimated. The contributions from these different effects are summarized in Table 1 . The total systematic uncertainties on the $\mathrm{a}_{1} / \mathrm{a}_{2}, \tilde{\mathrm{g}}_{\mathrm{M} 1}$ and $\mathrm{g}_{\mathrm{P}}$ parameters are, respectively, $\pm 0.02 \mathrm{GeV}^{2}, \pm 0.07$ and \pm 0.02 , yielding:

$$
\begin{gathered}
\mathrm{a}_{1} / \mathrm{a}_{2}=\left(-0.81_{-0.13 \text { stat }}^{+0.07} \pm 0.02_{\text {syst }}\right) \mathrm{GeV}^{2} \\
\tilde{\mathrm{g}}_{\mathrm{M} 1}=0.99_{-0.27 \text { stat }}^{+0.28} \pm 0.07_{\text {syst }} \\
\mathrm{g}_{\mathrm{P}}=0.19 \pm 0.04_{\text {stat }} \pm 0.02_{\text {syst }} .
\end{gathered}
$$

From the $g_{P}$ value above, we obtain a measurement of the charge radius of the neutral kaon:

$$
<\mathrm{R}^{2}>_{K^{0}}=-0.090 \pm 0.021 \mathrm{fm}^{2},
$$

not inconsistent with existing measurements obtained from coherent regeneration of short-lived neutral kaons by atomic electrons [43, 44, 45].

Using the fitted values of $a_{1} / a_{2}, \tilde{g}_{M 1}$ and $g_{P}$ as input parameters to the Monte Carlo simulation, the acceptance for $K_{\mathrm{L}} \rightarrow \pi^{+} \pi^{-} e^{+} e^{-}$decays is found to be $\left(3.49 \pm 0.01_{\text {stat }}\right) \%$. Tables 2 and 3 summarize, for the $K_{\mathrm{L}}$ and $K_{\mathrm{S}}$ data samples respectively, the $\pi^{+} \pi^{-} e^{+} e^{-}$and $K_{\mathrm{L}} \rightarrow \pi^{+} \pi^{-} \pi_{\mathrm{D}}^{0}$ yields after background subtraction, together with the corresponding acceptances. 
$\operatorname{BR}\left(K_{\mathrm{L}, \mathrm{S}} \rightarrow \pi^{+} \pi^{-} e^{+} e^{-}\right)$

The branching ratios of $K_{\mathrm{L}, \mathrm{S}} \rightarrow \pi^{+} \pi^{-} e^{+} e^{-}$decays normalized to the one of the $K_{\mathrm{L}} \rightarrow$ $\pi^{+} \pi^{-} \pi_{\mathrm{D}}^{0}$ channel can be written as:

$$
\frac{\mathrm{BR}\left(K_{\mathrm{L}, \mathrm{S}} \rightarrow \pi^{+} \pi^{-} e^{+} e^{-}\right)}{\mathrm{BR}\left(K_{\mathrm{L}} \rightarrow \pi^{+} \pi^{-} \pi_{\mathrm{D}}^{0}\right)}=\frac{\mathrm{N}_{\pi \pi \mathrm{ee}}^{\mathrm{L}, \mathrm{S}}}{\mathrm{N}_{\pi \pi \pi_{\mathrm{D}}^{0}}^{\mathrm{L}, \mathrm{S}}} \frac{\mathrm{A}_{\pi \pi \pi_{\mathrm{D}}^{0}}^{\mathrm{L}, \mathrm{S}}}{\mathrm{A}_{\pi \pi \mathrm{ee}}^{\mathrm{L}, \mathrm{S}}} \mathrm{R}_{\epsilon}^{\mathrm{L}, \mathrm{S}} \mathrm{R}_{K}^{\mathrm{L}, \mathrm{S}}
$$

where $\mathrm{N}_{\pi \pi e e}$ and $\mathrm{N}_{\pi \pi \pi_{\mathrm{D}}^{0}}$ are, respectively, the signal and normalization yields obtained after background subtraction, $\mathrm{A}_{\pi \pi e e}$ and $\mathrm{A}_{\pi \pi \pi_{\mathrm{D}}^{0}}$ are the corresponding acceptances, $\mathbf{R}_{\epsilon}=\epsilon_{\pi \pi \pi_{\mathrm{D}}^{0}} / \epsilon_{\pi \pi e e}$ is the relative normalization to signal trigger efficiency, and $\mathrm{R}_{K}$ is the ratio, between normalization and signal channels, of kaons decaying in the fiducial region. The superscripts L,S denote the corresponding kaon production targets.

For the $K_{\mathrm{L}}$ mode, $\mathrm{R}_{K}^{\mathrm{L}}$ is equal to 1 . For $K_{\mathrm{S}}$ data, the fraction of $K_{\mathrm{L}}$ to decaying $K_{\mathrm{S}}$ in the fiducial region depends on both the kaon production spectrum and the $K_{\mathrm{L}}-K_{\mathrm{S}}$ lifetime difference. Based on a Monte Carlo calculation, the fractions of kaons decaying in the fiducial region with respect to those produced at the $K_{\mathrm{S}}$ target are estimated to be $26.11 \%$ for $K_{\mathrm{S}}$ and $3.46 \%$ for $K_{\mathrm{L}}$, yielding the value $\mathrm{R}_{\mathrm{K}}^{\mathrm{S}}=0.133 \pm 0.002$. The uncertainty on $\mathrm{R}_{K}^{\mathrm{S}}$ is mainly due to the error in the kaon spectrum determination.

Since the topologies of $K_{\mathrm{L}, \mathrm{S}} \rightarrow \pi^{+} \pi^{-} e^{+} e^{-}$and $K_{\mathrm{L}} \rightarrow \pi^{+} \pi^{-} \pi_{\mathrm{D}}^{0}$ events in the magnetic spectrometer are very similar, the difference in trigger efficiency $\Delta \epsilon=\epsilon_{\pi \pi \pi_{\mathrm{D}}^{0}}-\epsilon_{\pi \pi e e}$ is expected to be small. To a good approximation, $\mathrm{R}_{\epsilon} \approx 1+\Delta \epsilon / \epsilon_{\pi \pi \pi_{\mathrm{D}}^{0}}$. As discussed in Sect. 4, the 4-track trigger efficiency has been measured using the abundant $K_{\mathrm{L}} \rightarrow \pi^{+} \pi^{-} \pi_{\mathrm{D}}^{0}$ events. The algorithm trigger efficiency, averaged over the 1998 and 1999 run periods, is $(97.9 \pm 0.1) \%$, in excellent agreement with the value of $98.0 \%$ obtained from the trigger simulation. As the numbers of $K_{\mathrm{L}, \mathrm{S}} \rightarrow \pi^{+} \pi^{-} e^{+} e^{-}$decays recorded with the downscaled control triggers are too small to provide an accurate measurement of the 4-track trigger efficiency, we rely on the simulation to extract $\Delta \epsilon$. We obtain the values $\Delta \epsilon^{\mathrm{L}}=(-0.3 \pm 0.1) \%$ for the data taken with the $K_{\mathrm{L}}$ beam, and $\Delta \epsilon^{\mathrm{S}}=(0.5 \pm 0.1) \%$ for the $K_{\mathrm{S}}$ data sample. Taking into account additional uncertainties due to trigger dead-time and the limited computation time available, we find $\mathrm{R}_{\epsilon}^{\mathrm{L}}=0.997 \pm 0.010$ and $\mathrm{R}_{\epsilon}^{\mathrm{S}}=1.005 \pm 0.003$.

The value of $\operatorname{BR}\left(K_{\mathrm{L}} \rightarrow \pi^{+} \pi^{-} \pi_{\mathrm{D}}^{0}\right)$, computed from the existing measurements of $\operatorname{BR}\left(K_{\mathrm{L}} \rightarrow \pi^{+} \pi^{-} \pi^{0}\right)$ and $\mathrm{BR}\left(\pi^{0} \rightarrow e^{+} e^{-} \gamma\right)$ [40], is $(1.505 \pm 0.047) \times 10^{-3}$. Using the yields and acceptances given in Tables 2 and 3, together with the values of $\mathbf{R}_{K}$ and $\mathbf{R}_{\epsilon}$ discussed above, the branching ratio $\mathrm{BR}\left(K_{\mathrm{L}} \rightarrow \pi^{+} \pi^{-} e^{+} e^{-}\right)$is found to be $\left(3.08 \pm 0.09_{\text {stat }}\right) \times 10^{-7}$ for the $1998+1999$ data, while for the $K_{\mathrm{S}}$ mode, the value $\operatorname{BR}\left(K_{\mathrm{S}} \rightarrow \pi^{+} \pi^{-} e^{+} e^{-}\right)=(4.71 \pm$ $\left.0.23_{\text {stat }}\right) \times 10^{-5}$ is obtained for the 1999 data. The quoted uncertainties reflect the statistics of the signal and normalization samples only.

Several additional sources of systematic uncertainties in the determination of the branching ratios have been investigated. Effects from accidental activity in the detectors have been studied by using Monte Carlo events overlaid with random triggers recorded proportionally to beam intensity during the various run periods. The comparison of overlaid events with the original ones allows the amount of gains and losses after event reconstruction to be estimated in both the signal and the normalization channels. To take properly into account possible biases generated by accidental high-multiplicity events in the spectrometer, the mechanism of overflow appearance in the drift chamber read-out has also been simulated. The overall systematic uncertainty coming from these effects is estimated to be $\pm 0.5 \%$ for the $K_{\mathrm{L}}$ data and $\pm 0.6 \%$ for the $K_{\mathrm{S}}$ data.

The sensitivity of the branching ratio measurements to the acceptance and selection cri- 
Table 4: Contributions to the relative systematic uncertainties in $\operatorname{BR}\left(K_{\mathrm{L}} \rightarrow \pi^{+} \pi^{-} e^{+} e^{-}\right)$and $\operatorname{BR}\left(K_{\mathrm{S}} \rightarrow \pi^{+} \pi^{-} e^{+} e^{-}\right)$.

\begin{tabular}{lcc}
\hline Source & $K_{\mathrm{L}}$ & $K_{\mathrm{S}}$ \\
& $(\%)$ & $(\%)$ \\
\hline MC statistics & \pm 0.4 & \pm 0.6 \\
Background & \pm 0.6 & \pm 0.3 \\
Accidental activity & \pm 0.5 & \pm 0.6 \\
Model and form factor & \pm 4.1 & - \\
Acceptance & \pm 2.2 & \pm 3.0 \\
Trigger & \pm 1.0 & \pm 0.3 \\
Kaon spectrum & \pm 0.1 & \pm 1.5 \\
\hline Total & \pm 4.8 & \pm 3.5 \\
\hline $\mathrm{BR}\left(K_{\mathrm{L}} \rightarrow \pi^{+} \pi^{-} \pi_{\mathrm{D}}^{0}\right)$ & \pm 3.1 & \pm 3.1 \\
\hline
\end{tabular}

teria has been checked by varying moderately the geometry and analysis cuts. Systematic uncertainties in the branching ratio of $\pm 2.2 \%$ and $\pm 3.0 \%$ have been estimated for the $K_{\mathrm{L}} \rightarrow$ $\pi^{+} \pi^{-} e^{+} e^{-}$and $K_{\mathrm{S}} \rightarrow \pi^{+} \pi^{-} e^{+} e^{-}$decay modes, respectively. In the case of the $K_{\mathrm{L}} \rightarrow \pi^{+} \pi^{-} e^{+} e^{-}$ channel, a large fraction of the total systematic uncertainty is due to the uncertainties in the form factor parameters. The $68.3 \%$ CL likelihood contour in the $\mathrm{a}_{1} / \mathrm{a}_{2}-\tilde{\mathrm{g}}_{\mathrm{M} 1}$ plane corresponds to a $\pm 4.0 \%$ variation in the $\mathrm{A}_{\pi \pi e e}$ acceptance value. Contributions from the uncertainty in $\mathrm{g}_{\mathrm{P}}$ and in the other parameters of the model are found to be less than $1 \%$.

Table 4 summarizes the contributions from the various sources of systematic uncertainties in the determination of $\operatorname{BR}\left(K_{\mathrm{L}} \rightarrow \pi^{+} \pi^{-} e^{+} e^{-}\right)$and $\mathrm{BR}\left(K_{\mathrm{S}} \rightarrow \pi^{+} \pi^{-} e^{+} e^{-}\right)$. Their sums, taken in quadrature, give total systematic uncertainties of $\pm 4.8 \%$ for the $K_{\mathrm{L}}$ mode and of $\pm 3.5 \%$ for the $K_{\mathrm{S}}$ mode. The uncertainty in the $\mathrm{BR}\left(K_{\mathrm{L}} \rightarrow \pi^{+} \pi^{-} \pi_{\mathrm{D}}^{0}\right)$ value brings an additional contribution of $\pm 3.1 \%$.

Based on the data collected in 1998 and 1999, the branching ratio for the $K_{\mathrm{L}} \rightarrow \pi^{+} \pi^{-} e^{+} e^{-}$ decay mode is measured to be $\operatorname{BR}\left(K_{\mathrm{L}} \rightarrow \pi^{+} \pi^{-} e^{+} e^{-}\right)=\left(3.08 \pm 0.09_{\text {stat }} \pm 0.15_{\text {syst }} \pm 0.10_{\text {norm }}\right) \times$ $10^{-7}$. Summing in quadrature the statistical, systematic and normalization uncertainties, we find:

$$
\operatorname{BR}\left(K_{\mathrm{L}} \rightarrow \pi^{+} \pi^{-} e^{+} e^{-}\right)=(3.08 \pm 0.20) \times 10^{-7},
$$

in agreement with the predictions of Refs. [7, 8].

For the $K_{\mathrm{S}} \rightarrow \pi^{+} \pi^{-} e^{+} e^{-}$mode, using the 1999 data samples, we measure $\mathrm{BR}\left(K_{\mathrm{S}} \rightarrow\right.$ $\left.\pi^{+} \pi^{-} e^{+} e^{-}\right)=\left(4.71 \pm 0.23_{\text {stat }} \pm 0.16_{\text {syst }} \pm 0.15_{\text {norm }}\right) \times 10^{-5}$, yielding:

$$
\operatorname{BR}\left(K_{\mathrm{S}} \rightarrow \pi^{+} \pi^{-} e^{+} e^{-}\right)=(4.71 \pm 0.32) \times 10^{-5} .
$$

Combining this result with the published value [14] of the branching ratio $\mathrm{BR}\left(K_{\mathrm{S}} \rightarrow \pi^{+} \pi^{-} e^{+} e^{-}\right)$ measured with the data collected in 1998, we obtain:

$$
\mathrm{BR}\left(K_{\mathrm{S}} \rightarrow \pi^{+} \pi^{-} e^{+} e^{-}\right)=(4.69 \pm 0.30) \times 10^{-5} .
$$

The latter result can be used to determine the $\mathrm{CP}$ violating inner bremsstrahlung part of the $K_{\mathrm{L}} \rightarrow \pi^{+} \pi^{-} e^{+} e^{-}$branching ratio through the relation:

$$
\frac{\mathrm{BR}\left(K_{\mathrm{L}}^{\mathrm{IB}} \rightarrow \pi^{+} \pi^{-} e^{+} e^{-}\right)}{\operatorname{BR}\left(K_{\mathrm{S}} \rightarrow \pi^{+} \pi^{-} e^{+} e^{-}\right)}=\frac{\tau_{\mathrm{L}}}{\tau_{\mathrm{S}}}\left|\eta_{+-}\right|^{2} .
$$


From Eqs. (13) and (14), and using the current experimental values for $\tau_{\mathrm{S}}, \tau_{\mathrm{L}}$ and $\eta_{+-}$[40], we find:

$$
\mathrm{BR}\left(K_{\mathrm{L}}^{\mathrm{IB}} \rightarrow \pi^{+} \pi^{-} e^{+} e^{-}\right)=(1.40 \pm 0.09) \times 10^{-7},
$$

in accord with theoretical predictions [7, 8]. Similarly, one can determine for the $K_{\mathrm{L}} \rightarrow$ $\pi^{+} \pi^{-} e^{+} e^{-}$process, the ratio of the $\mathrm{CP}$-violating part to the $\mathrm{CP}$-conserving one in the decay rate:

$$
\frac{\Gamma\left(K_{\mathrm{L}} \rightarrow \pi^{+} \pi^{-} e^{+} e^{-}\right)^{\mathrm{CPV}}}{\Gamma\left(K_{\mathrm{L}} \rightarrow \pi^{+} \pi^{-} e^{+} e^{-}\right)^{\mathrm{CPC}}}=0.833 \pm 0.066
$$

The measured values of $\operatorname{BR}\left(K_{\mathrm{L}} \rightarrow \pi^{+} \pi^{-} e^{+} e^{-}\right)$and $\mathrm{BR}\left(K_{\mathrm{S}} \rightarrow \pi^{+} \pi^{-} e^{+} e^{-}\right)$with their statistical uncertainty are shown in Fig. 11 for the different run periods.

\section{$9 \quad \mathcal{A}_{\phi}^{\mathrm{L}, \mathrm{S}}$ asymmetries}

The measurement of the CP-violating asymmetry $\mathcal{A}_{\phi}^{\mathrm{L}, \mathrm{S}}$ in $K_{\mathrm{L}, \mathrm{S}} \rightarrow \pi^{+} \pi^{-} e^{+} e^{-}$decays can be obtained from the distribution of events in the $\sin \phi \cos \phi$ variable:

$$
\mathcal{A}_{\phi}=\frac{\mathrm{N}_{\pi \pi \mathrm{ee}}(\sin \phi \cos \phi>0)-\mathrm{N}_{\pi \pi \mathrm{ee}}(\sin \phi \cos \phi<0)}{\mathrm{N}_{\pi \pi \mathrm{ee}}(\sin \phi \cos \phi>0)+\mathrm{N}_{\pi \pi \mathrm{ee}}(\sin \phi \cos \phi<0)}
$$

with the quantity $\sin \phi \cos \phi$ defined as

$$
\sin \phi \cos \phi=\left(\hat{\mathrm{n}}_{\mathrm{ee}} \times \hat{\mathrm{n}}_{\pi \pi}\right) \cdot \hat{\mathrm{z}}\left(\hat{\mathrm{n}}_{\mathrm{ee}} \cdot \hat{\mathrm{n}}_{\pi \pi}\right) .
$$

In Eq. 18, $\hat{\mathrm{n}}_{\mathrm{ee}}$ and $\hat{\mathrm{n}}_{\pi \pi}$ are respectively the unit vectors normal to the $e^{+} e^{-}$and $\pi^{+} \pi^{-}$planes, and $\hat{z}$ is the unit vector in the $\pi^{+} \pi^{-}$momentum direction in the kaon centre-of-mass system. Inspection of Eq. 18 shows that $\sin \phi \cos \phi$ changes sign under $\mathrm{CP}$.

In the case of $K_{\mathrm{L}} \rightarrow \pi^{+} \pi^{-} e^{+} e^{-}$decays, large CP-violating effects are expected to show up in the $\phi$ distribution of events. Using the values of $a_{1} / a_{2}, \tilde{g}_{M 1}$ and $g_{P}$ determined from the data (see Sect. 7), the model of Ref. [8] predicts an asymmetry of $13.7 \%$ in the $\sin \phi \cos \phi$ variable. The asymmetry, however, is known to vary strongly over the entire phase-space of the $K_{\mathrm{L}} \rightarrow \pi^{+} \pi^{-} e^{+} e^{-}$decay. The corresponding asymmetry within the detector acceptance is computed to be $24.4 \%$. Such a significant enhancement results from the fact that the detector acceptance favours phase-space regions where the interference between the M1 direct emission and inner bremsstrahlung processes is more important. Fig. 12(a) shows, for the $1998+1999$ data samples, the distribution of $K_{\mathrm{L}} \rightarrow \pi^{+} \pi^{-} e^{+} e^{-}$events as a function of $\sin \phi \cos \phi$. The shape of the measured angular distribution is well reproduced by the theoretical model and exhibits a clear asymmetry between regions near $|\sin \phi \cos \phi|=0.5$. The value of the asymmetry is measured to be $\left(24.9 \pm 2.9_{\text {stat }}\right) \%$, in agreement with the Monte Carlo calculation.

The determination of $\mathcal{A}_{\phi}^{\mathrm{L}}$, averaged over the entire phase-space, is obtained by correcting the observed distribution of events with the computed detector acceptance shown in Fig. 12(b). The dependence of the acceptance has a slowly increasing behaviour as a function of $\sin \phi \cos \phi$, consistent with the enhancement of the asymmetry observed in the angular distribution of events. The measured $\mathcal{A}_{\phi}^{\mathrm{L}}$ asymmetry, obtained in a model-dependent way by taking into account the acceptance correction, is found to be $\left(14.2 \pm 3.0_{\text {stat }}\right) \%$ (see Fig. 12(c)).

In the case of $K_{\mathrm{S}} \rightarrow \pi^{+} \pi^{-} e^{+} e^{-}$decays, no asymmetry is observed in the $\sin \phi \cos \phi$ variable. Fig. 13(a) shows the corresponding angular distribution of the events after taking into account the acceptance correction. Since no interference term is present in the amplitude of this decay mode, the acceptance is found to be rather uniform over the entire $\sin \phi \cos \phi$ domain, exhibiting no asymmetric term in that variable (see Fig. 13(b)). Based on the data collected in 
Table 5: Contributions to the systematic uncertainties in $\mathcal{A}_{\phi}^{\mathrm{L}}$ and $\mathcal{A}_{\phi}^{\mathrm{S}}$.

\begin{tabular}{lcc}
\hline Source & $K_{\mathrm{L}}$ & $K_{\mathrm{S}}$ \\
& $(\%)$ & $(\%)$ \\
\hline MC statistics & \pm 0.3 & \pm 0.4 \\
Background & \pm 0.5 & \pm 0.3 \\
Accidental activity & \pm 0.3 & \pm 0.4 \\
Model and form factor & \pm 1.2 & - \\
Acceptance & \pm 1.2 & \pm 1.4 \\
Resolution & \pm 0.5 & \pm 0.5 \\
\hline Total & \pm 1.9 & \pm 1.6 \\
\hline
\end{tabular}

$1999, \mathcal{A}_{\phi}^{\mathrm{S}}$ is measured to be $\left(0.5 \pm 4.0_{\text {stat }}\right) \%$. This result demonstrates that the large value of $\mathcal{A}_{\phi}^{\mathrm{L}}$ observed in the $K_{\mathrm{L}} \rightarrow \pi^{+} \pi^{-} e^{+} e^{-}$decay mode cannot originate from asymmetries in the detector elements.

The systematic uncertainties affecting the measurement of $\mathcal{A}_{\phi}^{\mathrm{L}, \mathrm{S}}$ have been estimated in a similar way to that for the branching ratio determination. Their contributions are given in Table 5 for both $K_{\mathrm{L}}$ and $K_{\mathrm{S}}$ modes. For $K_{\mathrm{L}} \rightarrow \pi^{+} \pi^{-} e^{+} e^{-}$decays, the uncertainty in $\mathcal{A}_{\phi}^{\mathrm{L}}$ due to the model and form factor parameters used for the acceptance calculation, is estimated to be $\pm 1.2 \%$. This value is largely dominated by the statistical accuracy, given by the likelihood contour at $68 \% \mathrm{CL}$, in the determination of the $\mathrm{a}_{1} / \mathrm{a}_{2}$ and $\tilde{\mathrm{g}}_{\mathrm{M} 1}$ parameters. Resolution effects in the $\phi$ angle computation have also been taken into account by smearing the track parameters with resolution functions determined with the Monte Carlo simulation and by studying possible biases due to the residual magnetic field inside the kaon decay volume. The corresponding systematic uncertainty is estimated to be $\pm 0.5 \%$ for both $K_{\mathrm{L}}$ and $K_{\mathrm{S}}$ modes. The sums in quadrature of the various contributions to the systematic uncertainties are $\pm 1.9 \%$ in $\mathcal{A}_{\phi}^{\mathrm{L}}$ and $\pm 1.6 \%$ in $\mathcal{A}_{\phi}^{\mathrm{S}}$ (see Table 5).

From the data collected in the 1998 and 1999 runs, the asymmetry in the $K_{\mathrm{L}} \rightarrow \pi^{+} \pi^{-} e^{+} e^{-}$ mode is $\mathcal{A}_{\phi}^{\mathrm{L}}=\left(14.2 \pm 3.0_{\text {stat }} \pm 1.9_{\text {syst }}\right) \%$. Adding in quadrature the statistical and systematic uncertainties, we obtain:

$$
\mathcal{A}_{\phi}^{\mathrm{L}}=(14.2 \pm 3.6) \% \text {. }
$$

Our result is in agreement with the recent measurement of the KTeV experiment [12] and constitutes a clear signature of the presence of CP-violating effects in the decay mode $K_{\mathrm{L}} \rightarrow$ $\pi^{+} \pi^{-} e^{+} e^{-}$.

From the analysis of the $1999 K_{\mathrm{S}}$ samples, the asymmetry is measured to be $\mathcal{A}_{\phi}^{\mathrm{S}}=$ $\left(0.5 \pm 4.0_{\text {stat }} \pm 1.6_{\text {syst }}\right) \%$, yielding the value:

$$
\mathcal{A}_{\phi}^{\mathrm{S}}=(0.5 \pm 4.3) \% \text {. }
$$

Combining the published sample of $56 K_{\mathrm{S}} \rightarrow \pi^{+} \pi^{-} e^{+} e^{-}$events observed in 1998 [14] with the 1999 statistics, we obtain:

$$
\mathcal{A}_{\phi}^{\mathrm{S}}=(-1.1 \pm 4.1) \%
$$

consistent with zero. 


\section{Conclusions}

Using the data collected in 1998 and 1999 with the NA48 detector at CERN, we have performed precise measurements of the branching ratios and asymmetries for the $K_{\mathrm{L}} \rightarrow \pi^{+} \pi^{-} e^{+} e^{-}$ and $K_{\mathrm{S}} \rightarrow \pi^{+} \pi^{-} e^{+} e^{-}$decay modes. Our results are in good agreement with theoretical models based on a phenomenological description of radiative kaon decays. Manifestation of indirect CP violation in the decay channel $K_{\mathrm{L}} \rightarrow \pi^{+} \pi^{-} e^{+} e^{-}$has been confirmed by the measurement of a large asymmetry in the angular correlation between the $\pi^{+} \pi^{-}$and $e^{+} e^{-}$decay planes. No such asymmetry is observed for $K_{\mathrm{S}} \rightarrow \pi^{+} \pi^{-} e^{+} e^{-}$decays. The measured values of the $K^{0}$ charge radius and of the form factor parameters in the M1 direct emission process in $K_{\mathrm{L}} \rightarrow \pi^{+} \pi^{-} e^{+} e^{-}$ decays are consistent with published results.

\section{Acknowledgements}

We would like to thank the technical staff of the participating laboratories, universities and affiliated computing centres for their efforts in the construction of the NA48 detector, in the operation of the experiment and in the processing of the data.

\section{References}

[1] H. Chew, Nuovo Cimento 26, 1109-1127 (1962)

[2] G. Costa and P.K. Kabir, Nuovo Cimento A 51, 564-569 (1967)

[3] L.M. Sehgal and L. Wolfenstein, Phys. Rev. 162, 1362-1368 (1967)

[4] A.D. Dolgov and L.A. Ponomarev, Sov. J. Nucl. Phys. 4, 262-264 (1967)

[5] L.A. Kondratyuk, L.A. Ponomarev and V.I. Zacharov, Phys. Lett. 27 B, 655-656 (1968)

[6] D.P. Majumdar and J. Smith, Phys. Rev. 187, 2039-2043 (1969)

[7] L.M. Sehgal and M. Wanninger, Phys. Rev. D 46, 1035-1041 (1992); Erratum: Phys. Rev. D 46, 5209-5210 (1992)

[8] P. Heiliger and L.M. Sehgal, Phys. Rev. D 48, 4146-4154 (1993); Erratum: Phys. Rev. D 60, 079902 (1999)

[9] J.K. Elwood, M.B. Wise and M. Savage, Phys. Rev. D 52, 5095-5105 (1995)

[10] J.K. Elwood et al., Phys. Rev. D 53, 4078-4081 (1995)

[11] J. Adams et al., Phys. Rev. Lett. 80, 4123-4126 (1998)

[12] A. Alavi-Harati et al., Phys. Rev. Lett. 84, 408-411 (2000)

[13] Y. Takeuchi et al., Phys. Lett. B 443, 409-414 (1998)

[14] A. Lai et al., Phys. Lett. B 496, 137-144 (2000)

[15] J. Ellis and N.E. Mavromatos, Phys. Rept. 320, 341-354 (1999)

[16] L. Alvarez-Gaume et al., Proceedings of the XXXIVth Rencontres de Moriond, Les Arcs, France, March 13-20, 141-148 (1999)

[17] I.I. Bigi and A.I. Sanda, Phys. Lett. B 466, 33-40 (1999)

[18] L. Wolfenstein, Phys. Rev. Lett. 83, 911-912 (1999)

[19] J. van Leusen and L.M. Sehgal, Phys. Rev. Lett. 83, 49334396 (1999)

[20] J. van Leusen and L.M. Sehgal, Phys. Lett. B 489, 300-308 (2000)

[21] S.S. Bulanov, hep-ph/1099025, 27p. (2001) 
[22] G. Ecker and H. Pichl, Phys. Lett. B 507, 193-199 (2001)

[23] H. Pichl, Eur. Phys. J. C 20, 371-388 (2001)

[24] C. Biino et al., CERN-SL-98-041 EA and Proceedings of the 6th EPAC, Stockholm 1998, IoP, 2100-2102 (1999)

[25] N. Doble et al., Nucl. Instr. and Methods B 119, 181-191 (1996)

[26] A. Lai et al., Eur. Phys. J. C 22, 231-254 (2001)

[27] J.R. Batley et al., Phys. Lett. B 544, 97-112 (2002)

[28] D. Bédérède et al., Nucl. Instr. and Methods A 367, 88-91 (1995)

[29] M. Martini et al., Proc. 7th Int. Conf. on Calorimetry in

High Energy Physics, Tucson, AR, USA, World Scientific,

375-383 (1997)

[30] P. Grafström et al., Nucl. Instrum. Methods A 344, 487-491 (1994)

[31] H. Bergauer et al., Nucl. Instrum. Methods A 419, 623-631 (1998)

[32] S. Anvar et al., Nucl. Instrum. Methods A 419, 686-694 (1998)

[33] S. Anvar et al., Prepared for IEEE Nuclear Science Symposium (NSS) and Medical Imaging Conference (MIC), Toronto, Ontario, Canada, 8-14 Nov 1998, DAPNIA-99-06, 4p (1999)

[34] I. Augustin et al., Nucl. Instr. and Methods A 403, 472-480 (1998)

[35] R. Frühwirth, Nucl. Instr. and Methods A 262, 444-450 (1987)

[36] D. Luers et al., Phys. Rev. 133 B, 1276-1289 (1964)

[37] CERN Program Library Long Writeup W5013 (1993)

[38] E. Barbario, B. van Eijk and Z. Wa̧s, Comput. Phys. Commun. 66, 115-128 (1991); E. Barbario and Z. Wass, CERN-TH. 7033/93, 22p. (1993)

[39] K.O. Mikaelian and J. Smith, Phys. Rev. D 5, 2890-2895 (1972) and references therein

[40] Particle Data Group, Eur. Phys. J. C 15, 1-878 (2000)

[41] A. Alavi-Harati et al., Phys. Rev. Lett. 86, 761-764 (2001)

[42] CERN Program Library Long Writeup D506 (1998)

[43] H. Foeth et al., Phys. Lett. 30 B, 276-281 (1969)

[44] F. Dydak et al., Nucl. Phys. B 102, 253-274 (1976)

[45] W.R. Molzon et al., Phys. Rev. Lett. 41, 1213-1216 (1978) 


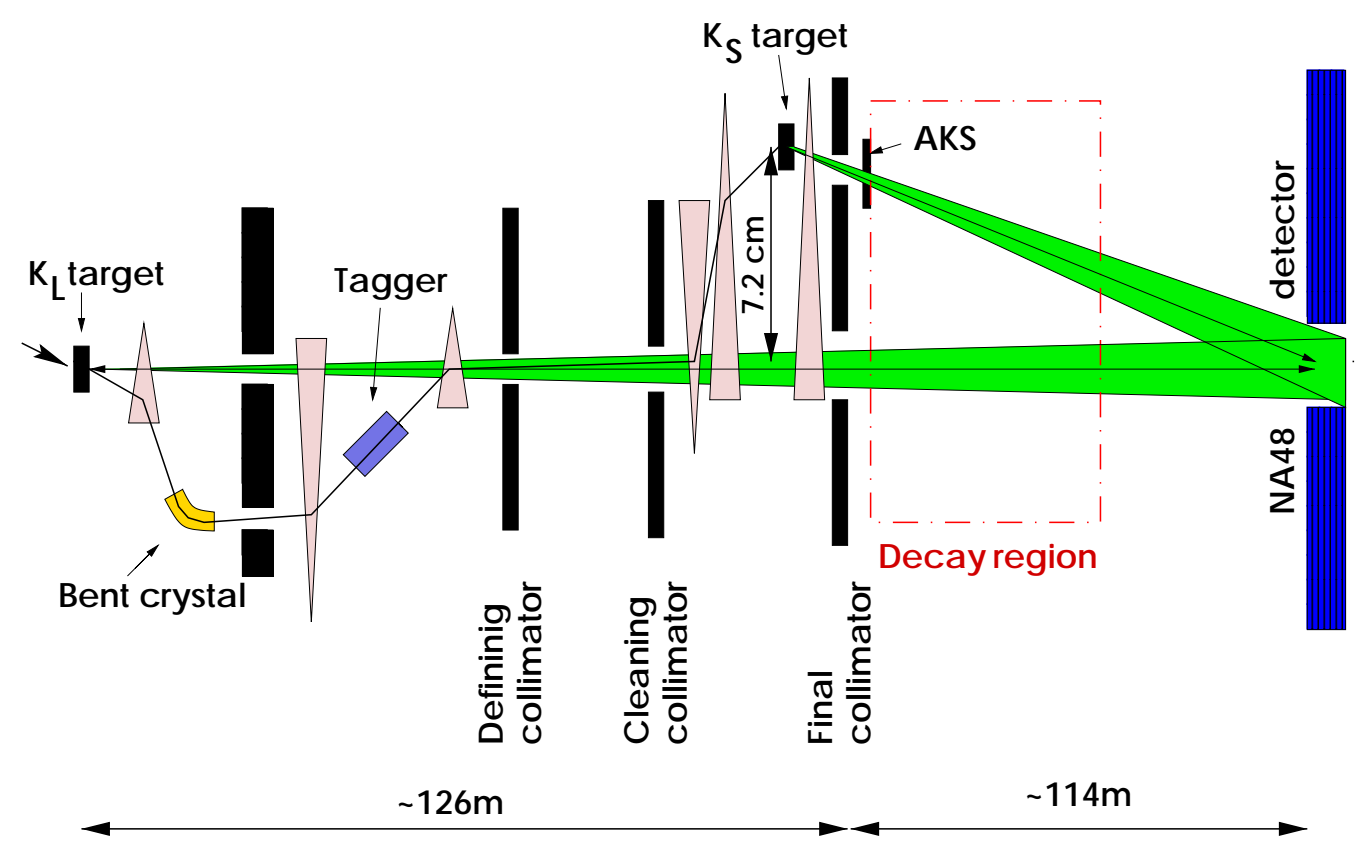

Figure 1: Schematic of the NA48 neutral kaon beams (not to scale).

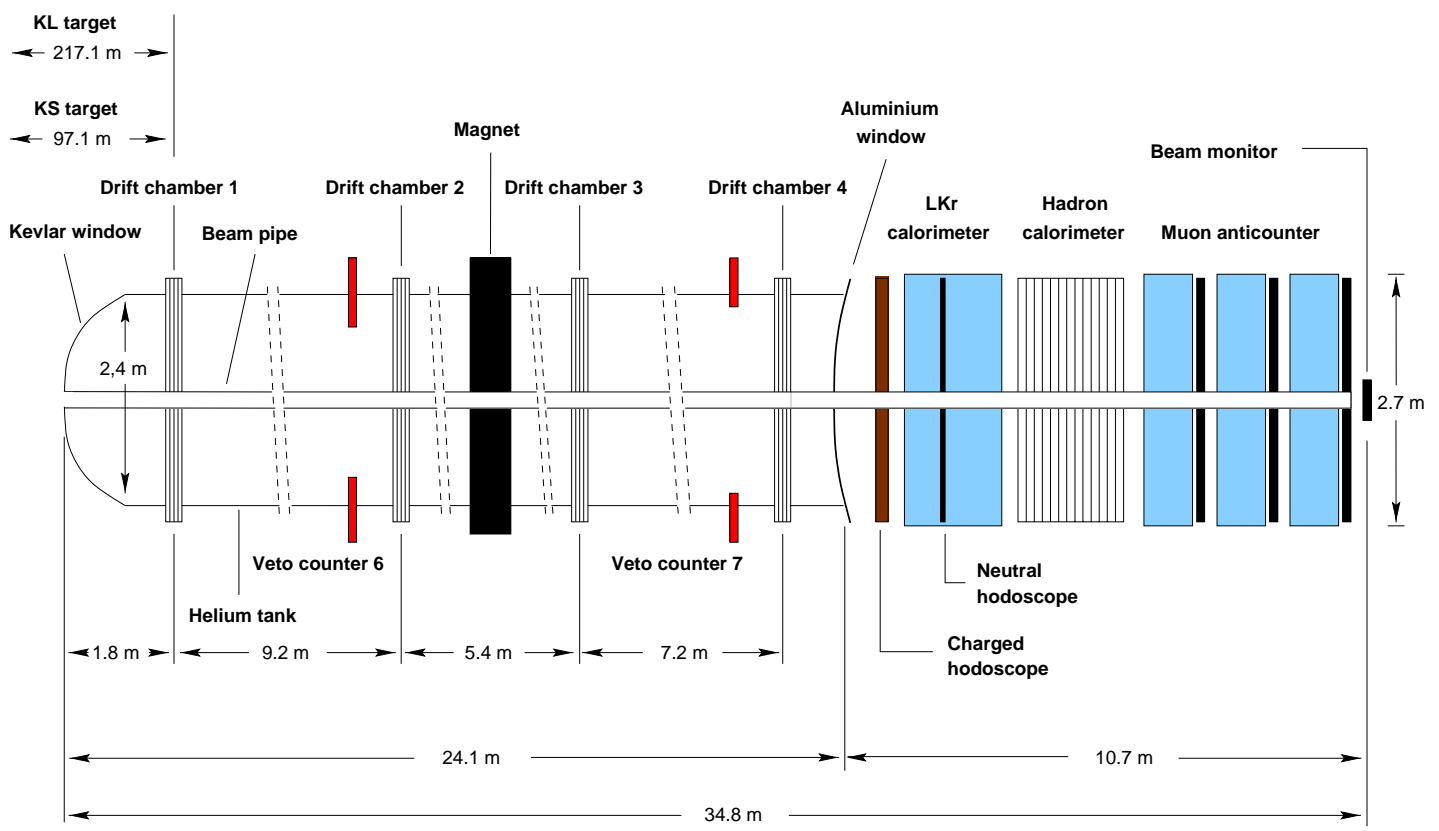

Figure 2: Layout of the NA48 detector. 


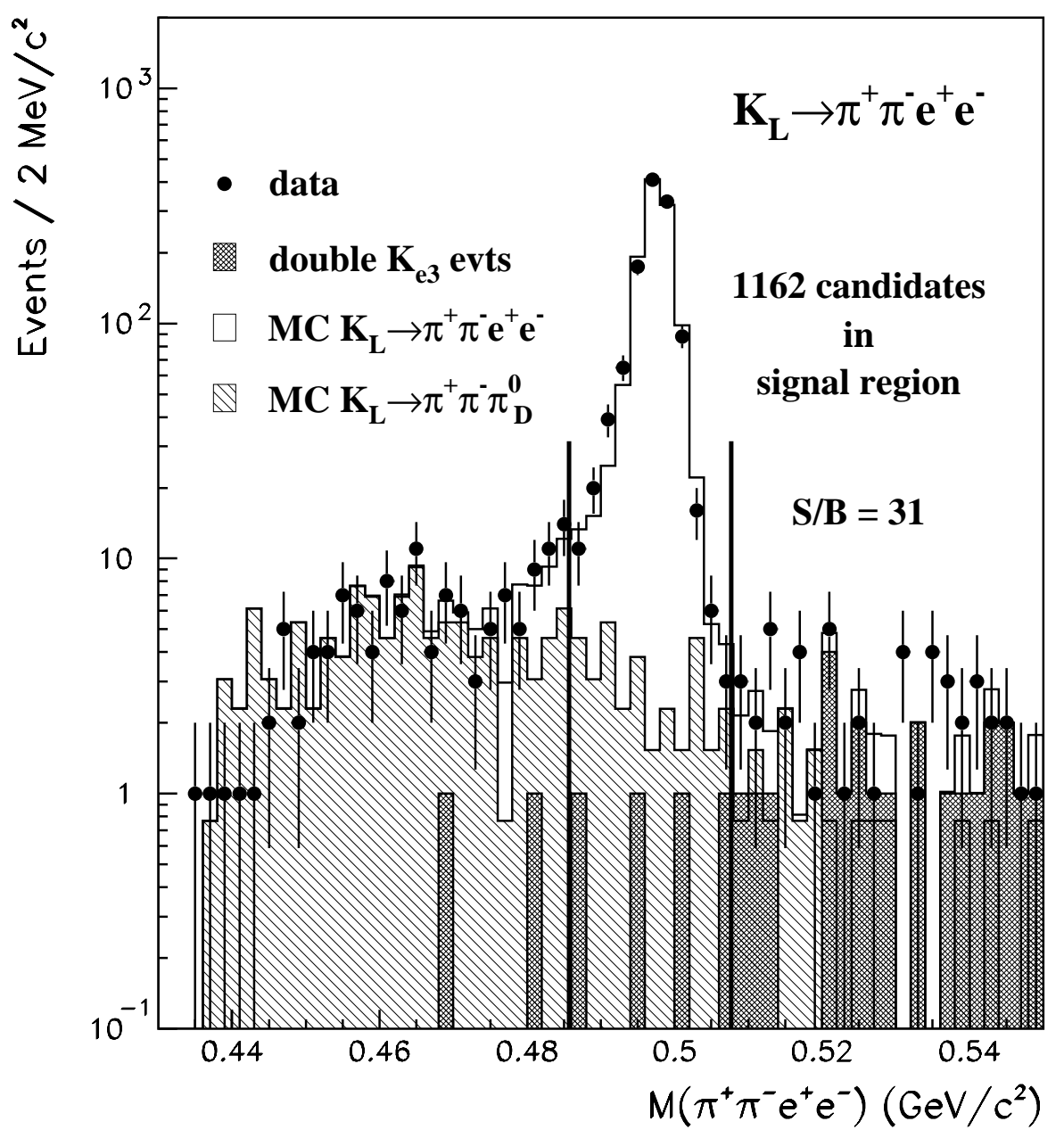

Figure 3: Invariant mass distribution $\mathrm{M}_{\pi \pi e e}$ for $K_{\mathrm{L}} \rightarrow \pi^{+} \pi^{-} e^{+} e^{-}$candidates. The signal region is indicated by thick lines. Data are represented by dots while the different histograms show the shape expected from Monte Carlo for good events and the background components due to $K_{\mathrm{L}} \rightarrow \pi^{+} \pi^{-} \pi_{\mathrm{D}}^{0}$ and overlapping $K_{\mathrm{e} 3}$ decays. 


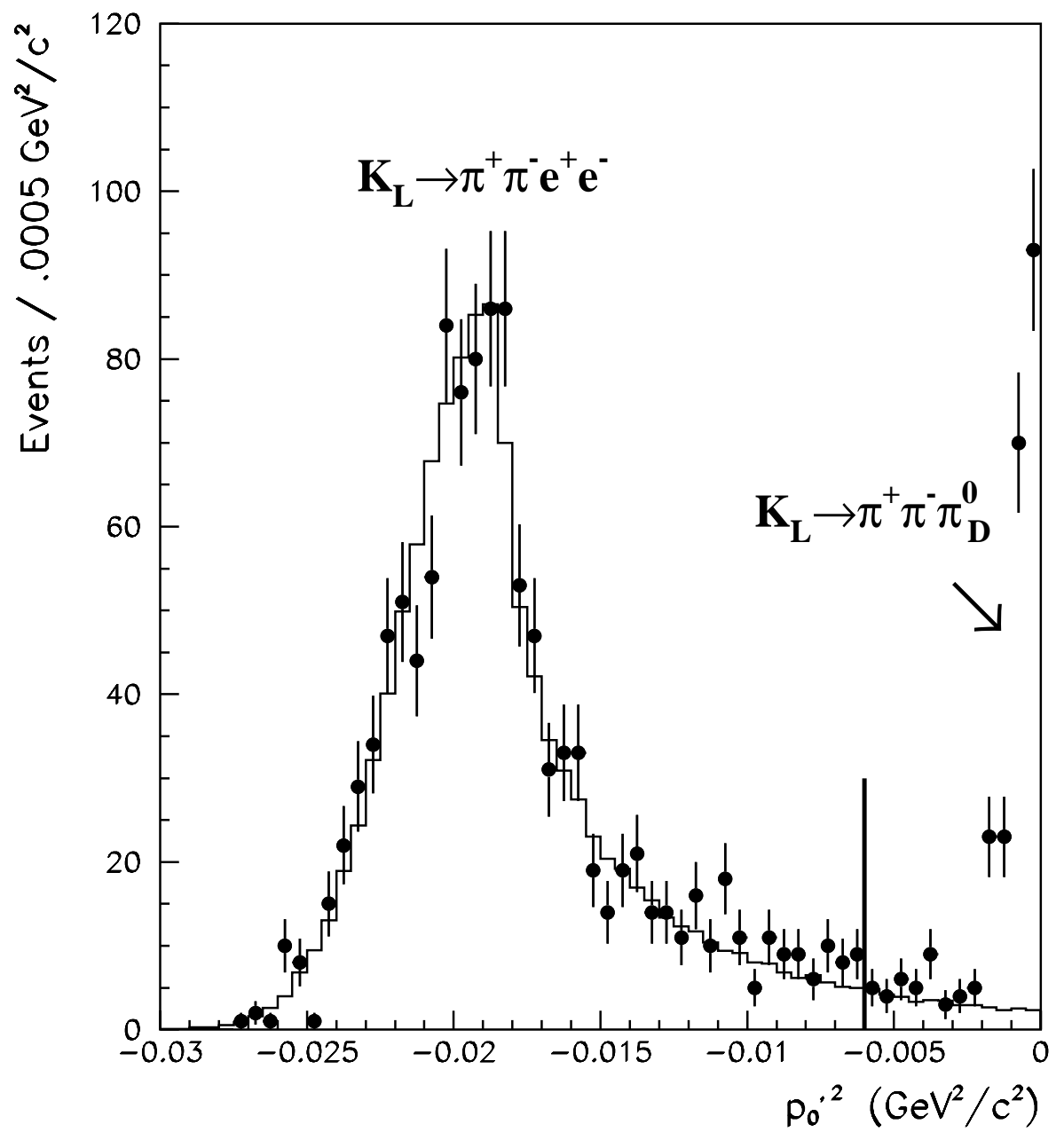

Figure 4: Distribution of events in the $\mathrm{p}_{0}^{\prime 2}$ variable for $K_{\mathrm{L}} \rightarrow \pi^{+} \pi^{-} e^{+} e^{-}$candidates (dots) after all other selection criteria have been applied. The histogram represents the Monte Carlo distribution for true $K_{\mathrm{L}} \rightarrow \pi^{+} \pi^{-} e^{+} e^{-}$events normalized to the number of observed good events. The tail near $\mathrm{p}_{0}^{\prime 2}=0$ is due to $K_{\mathrm{L}} \rightarrow \pi^{+} \pi^{-} \pi_{\mathrm{D}}^{0}$ decays. The signal region is limited by a thick line. 

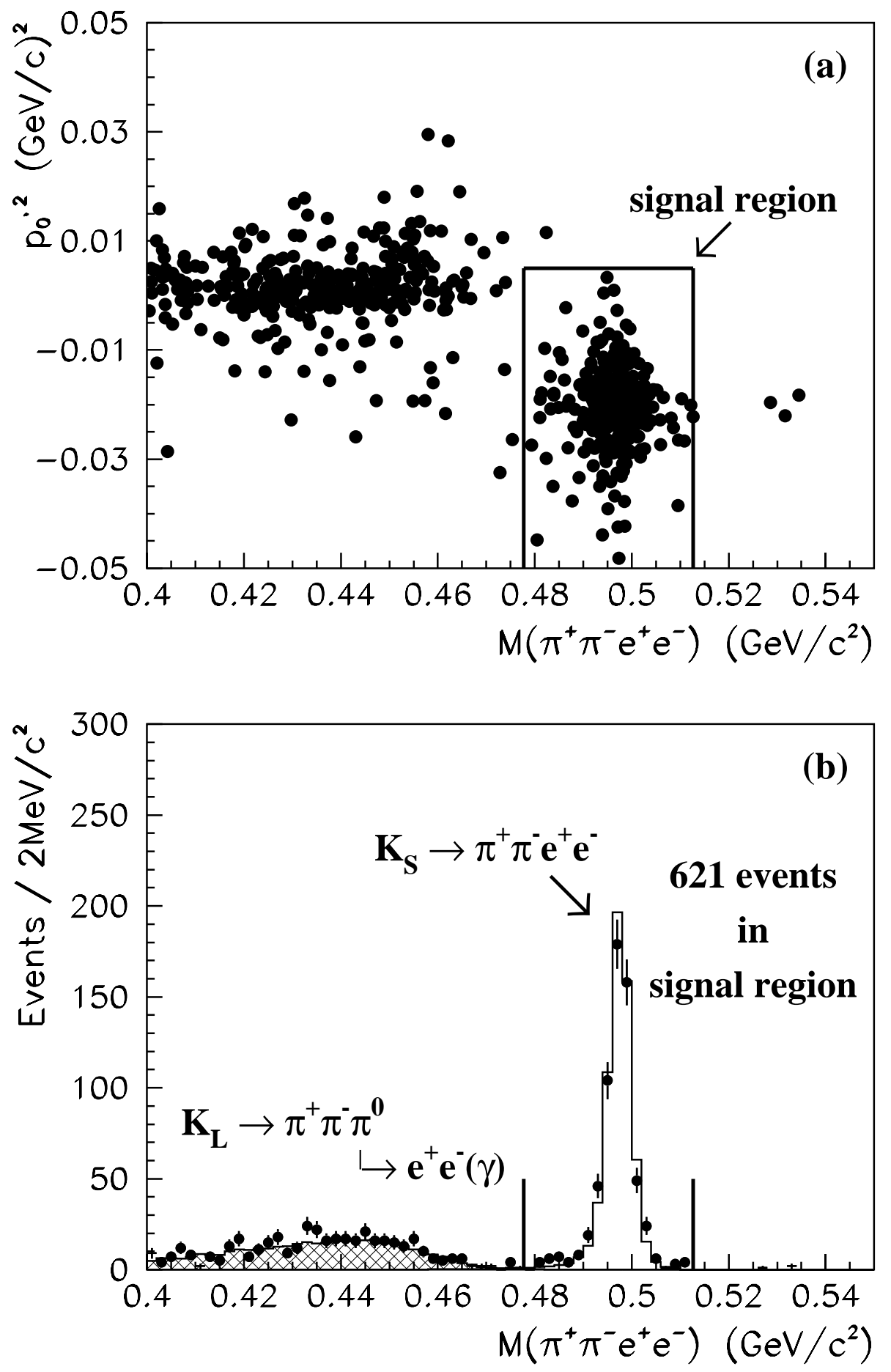

Figure 5: (a) $\mathrm{p}_{0}^{\prime 2}$ vs. $\mathrm{M}_{\pi \pi e e}$ scatter plots for $K_{\mathrm{S}} \rightarrow \pi^{+} \pi^{-} e^{+} e^{-}$candidates. The box indicates the signal region. (b) Invariant mass $\mathbf{M}_{\pi \pi e e}$ distribution of events above (dots). The histograms are the MC predictions for the signal (plain) and the $K_{\mathrm{L}} \rightarrow \pi^{+} \pi^{-} \pi_{\mathrm{D}}^{0}$ background events (hatched). 

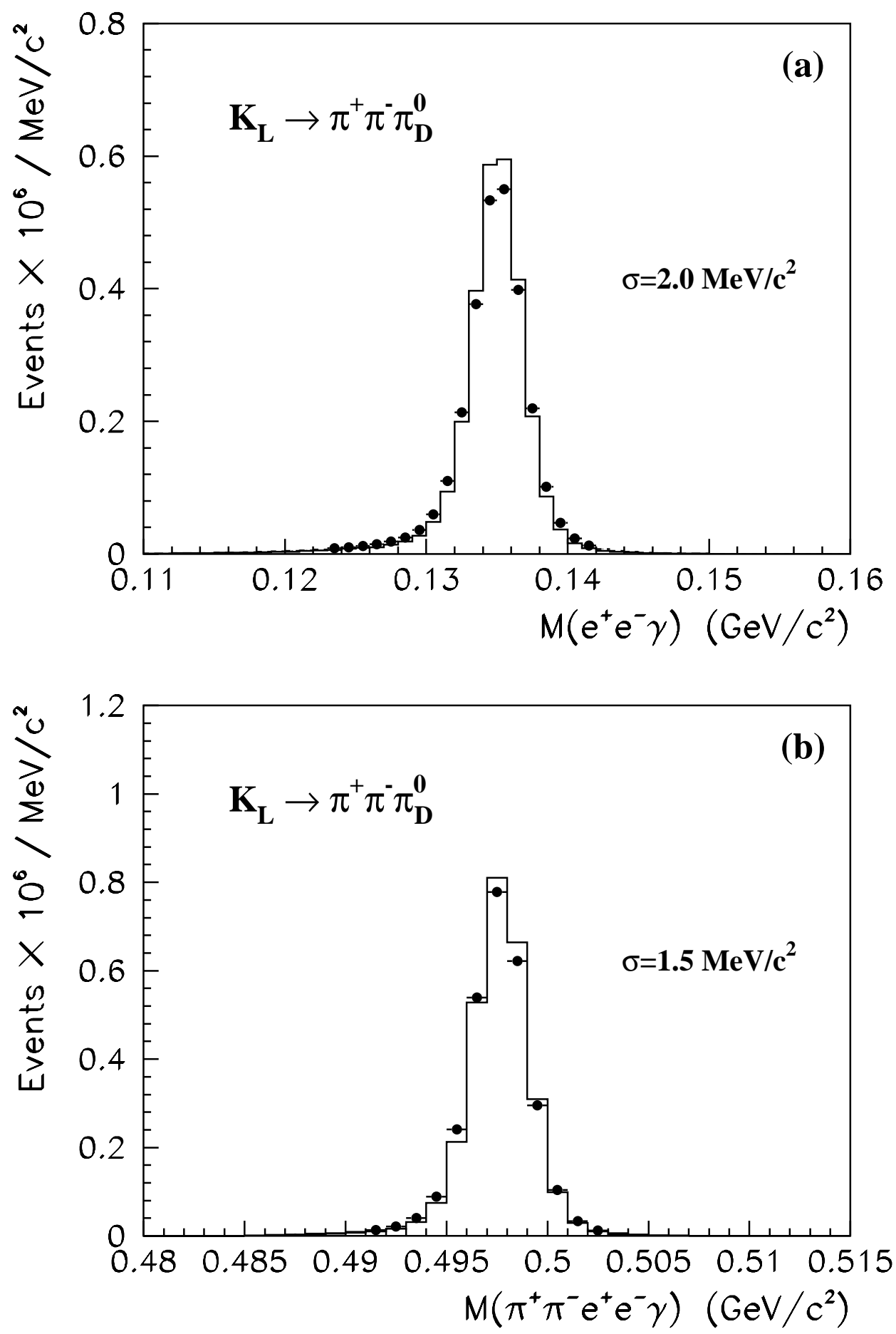

Figure 6: Invariant $\mathrm{M}_{e e \gamma}$ (a) and $\mathrm{M}_{\pi \pi e e \gamma}$ (b) mass distributions for $K_{\mathrm{L}} \rightarrow \pi^{+} \pi^{-} \pi_{\mathrm{D}}^{0}$ decays. Black dots represent data while the histograms are Monte Carlo predictions normalized to the total number of observed events. 

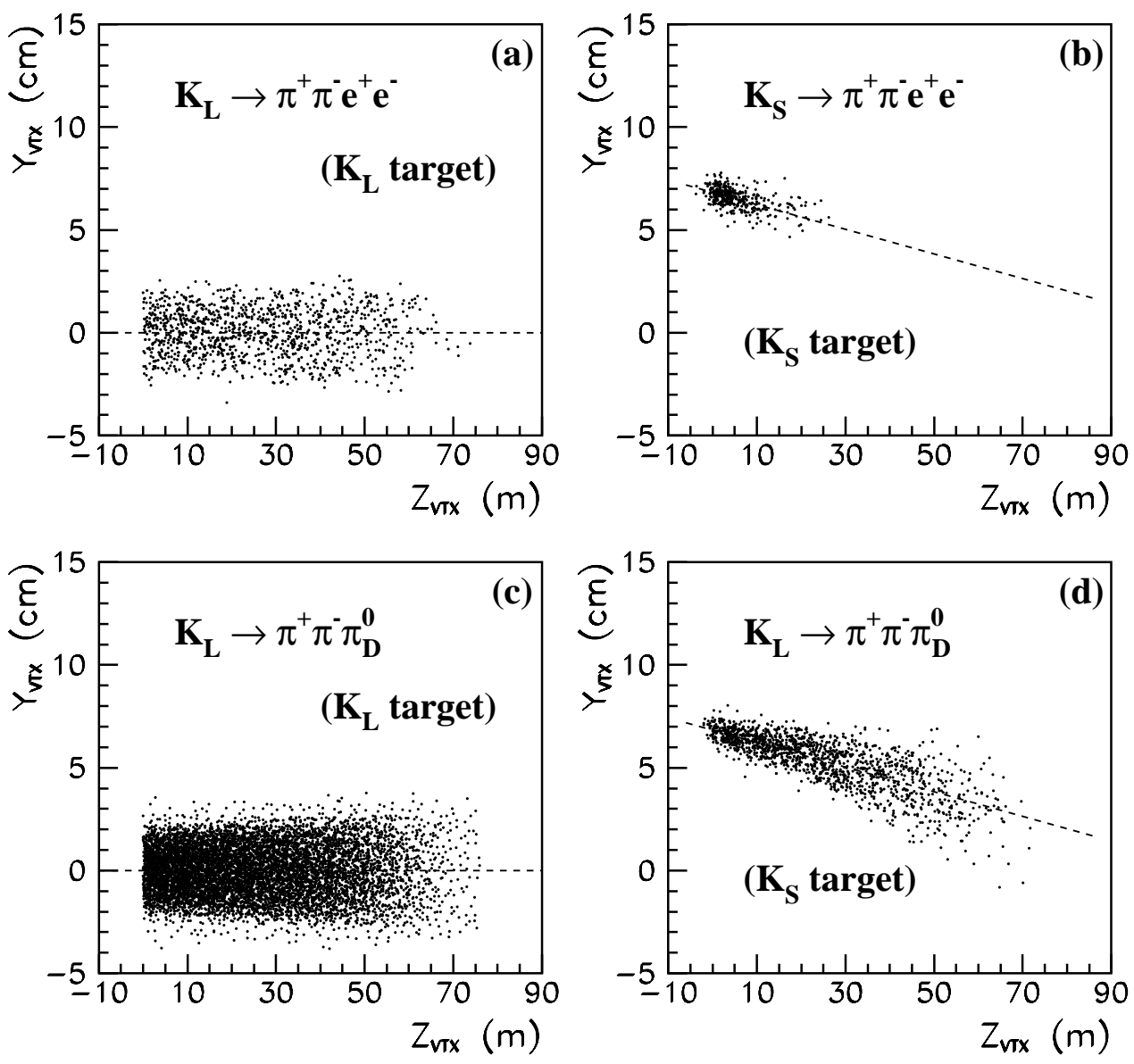

Figure 7: $\mathrm{Y}_{\mathrm{vtx}}$ vs. $\mathrm{Z}_{\mathrm{vtx}}$ scatter plots for identified $K_{\mathrm{L}} \rightarrow \pi^{+} \pi^{-} e^{+} e^{-}$(a) and $K_{\mathrm{S}} \rightarrow \pi^{+} \pi^{-} e^{+} e^{-}$ (b) events, as well as for $K_{\mathrm{L}} \rightarrow \pi^{+} \pi^{-} \pi_{\mathrm{D}}^{0}$ decays from the $K_{\mathrm{L}}$ target (c) and from the $K_{\mathrm{S}}$ target (d). The dashed lines shown in the scatter plots represent the respective nominal beam axes. 

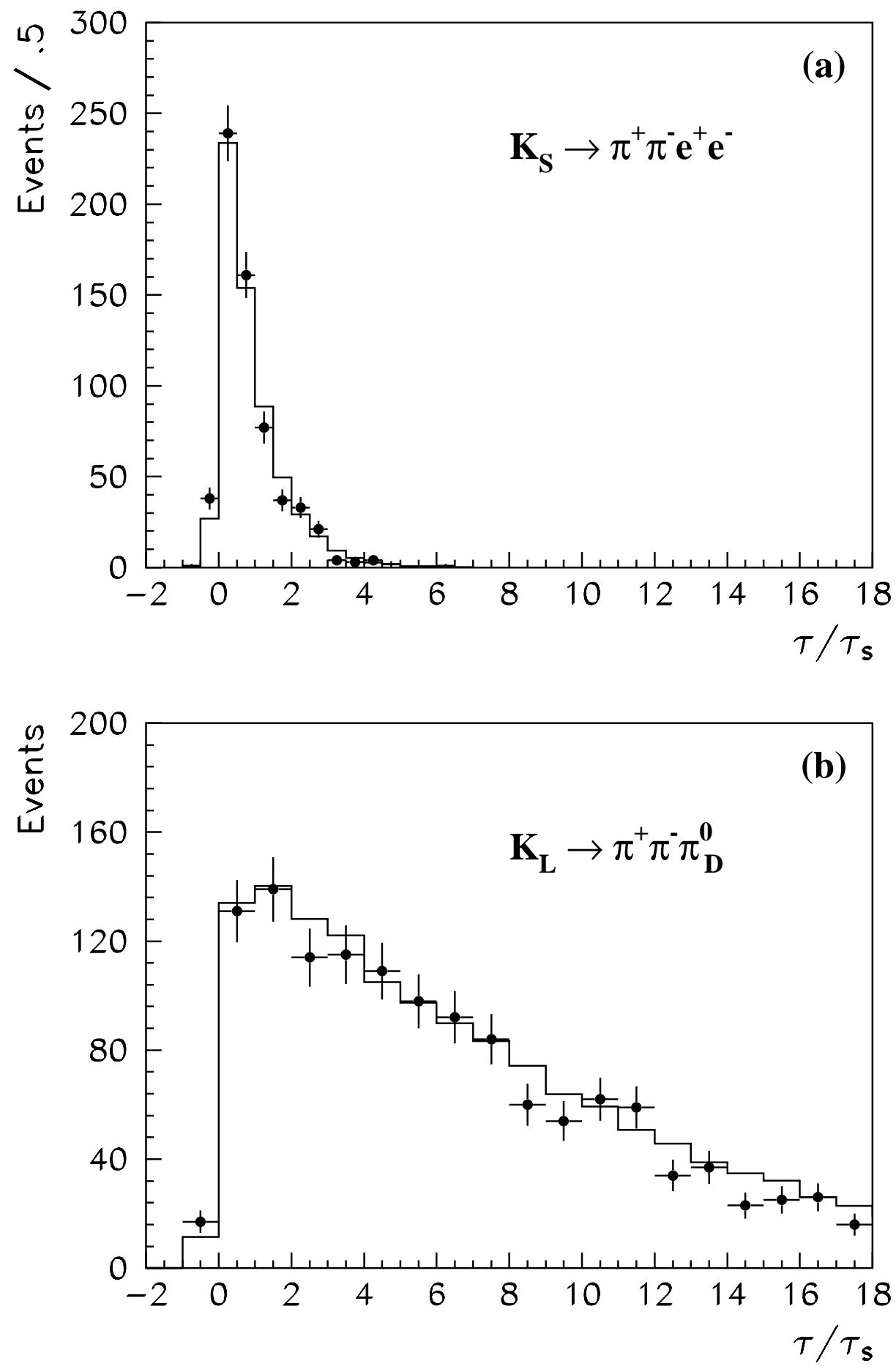

Figure 8: Proper decay time $\tau / \tau_{\mathrm{S}}$ distribution in units of the $K_{\mathrm{S}}$ lifetime for (a) the $K_{\mathrm{S}} \rightarrow$ $\pi^{+} \pi^{-} e^{+} e^{-}$mode and (b) the $K_{\mathrm{L}} \rightarrow \pi^{+} \pi^{-} \pi_{\mathrm{D}}^{0}$ mode. The histograms are the Monte Carlo distributions normalized to the corresponding observed numbers of events. 

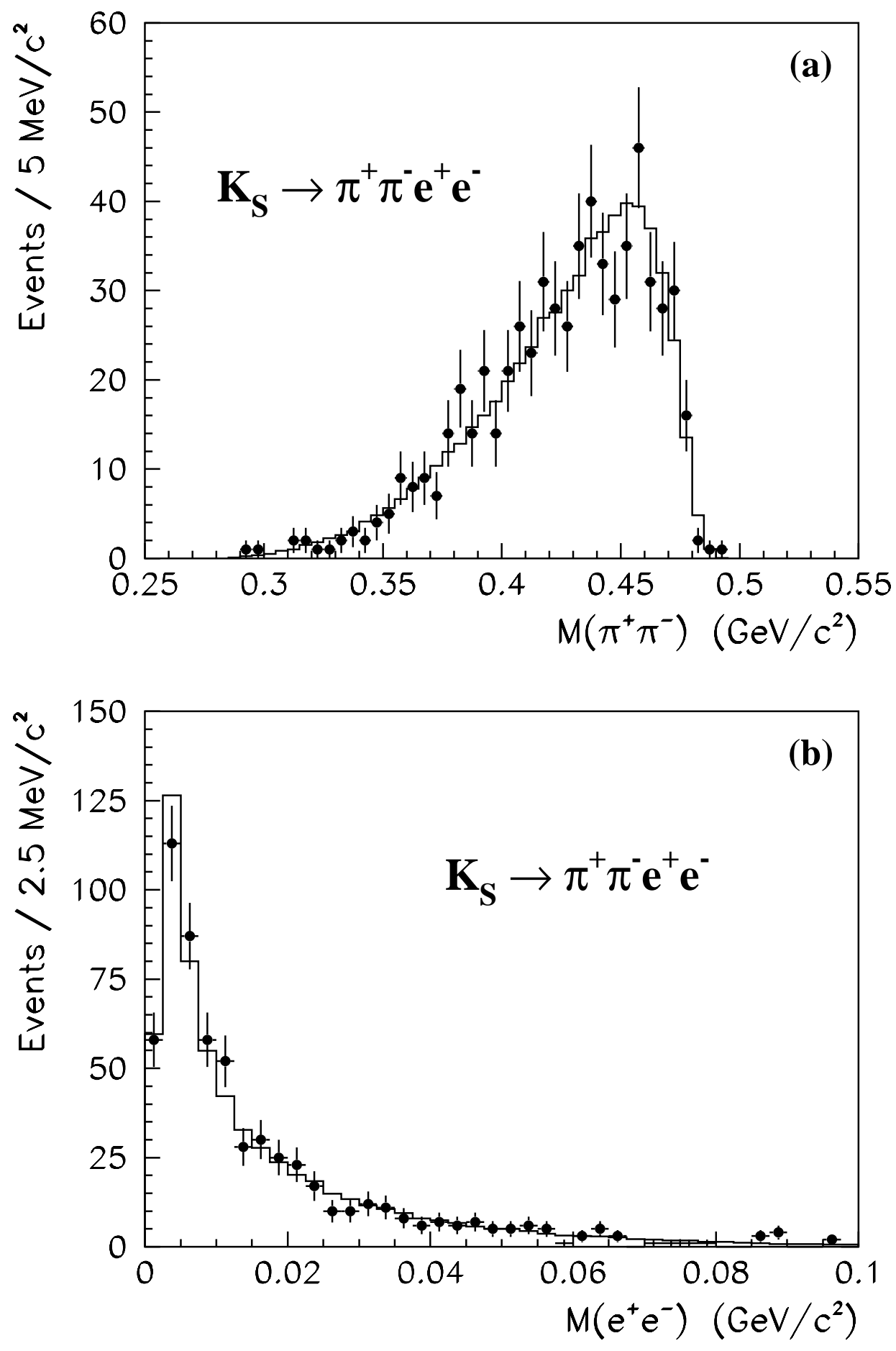

Figure 9: Invariant mass $\mathrm{M}_{\pi \pi}$ (a) and $\mathrm{M}_{e e}$ (b) distributions for $K_{\mathrm{S}} \rightarrow \pi^{+} \pi^{-} e^{+} e^{-}$decays. Data are shown as solid dots while the histograms are the Monte Carlo predictions normalized to the corresponding number of observed events 

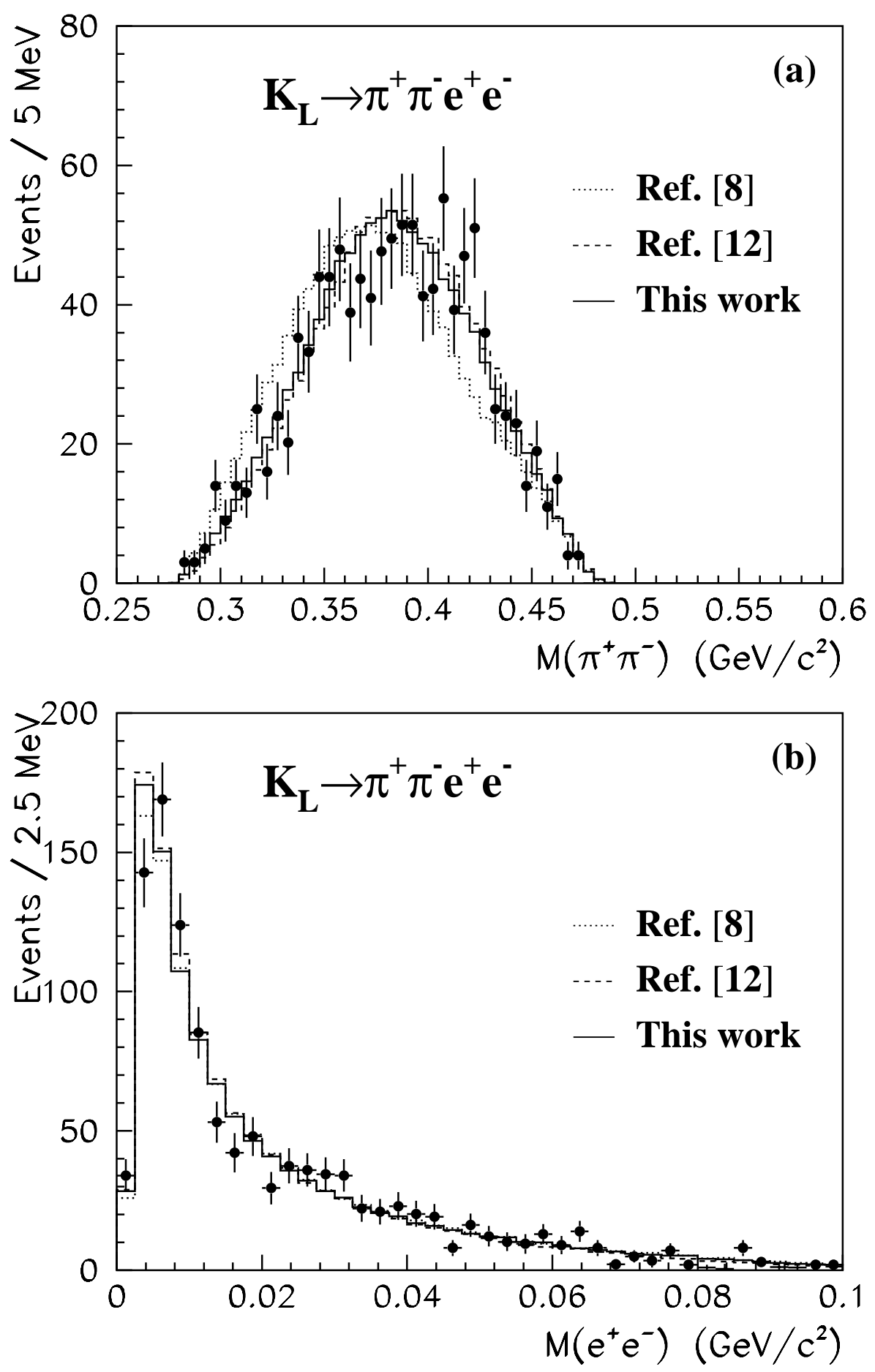

Figure 10: Invariant mass $\mathrm{M}_{\pi \pi}$ (a) and $\mathrm{M}_{e e}$ (b) distributions for $K_{\mathrm{L}} \rightarrow \pi^{+} \pi^{-} e^{+} e^{-}$decays. Data are shown with solid dots while the histograms are Monte Carlo predictions using different parameters for the form factor in the M1 direct emission amplitude and for the $K^{0}$ charge radius: dotted line from Ref. [8] (no form factor), dashed line from Ref. [12] (KTeV experiment) and solid line from this work. All histograms are normalized to the corresponding number of observed events. 

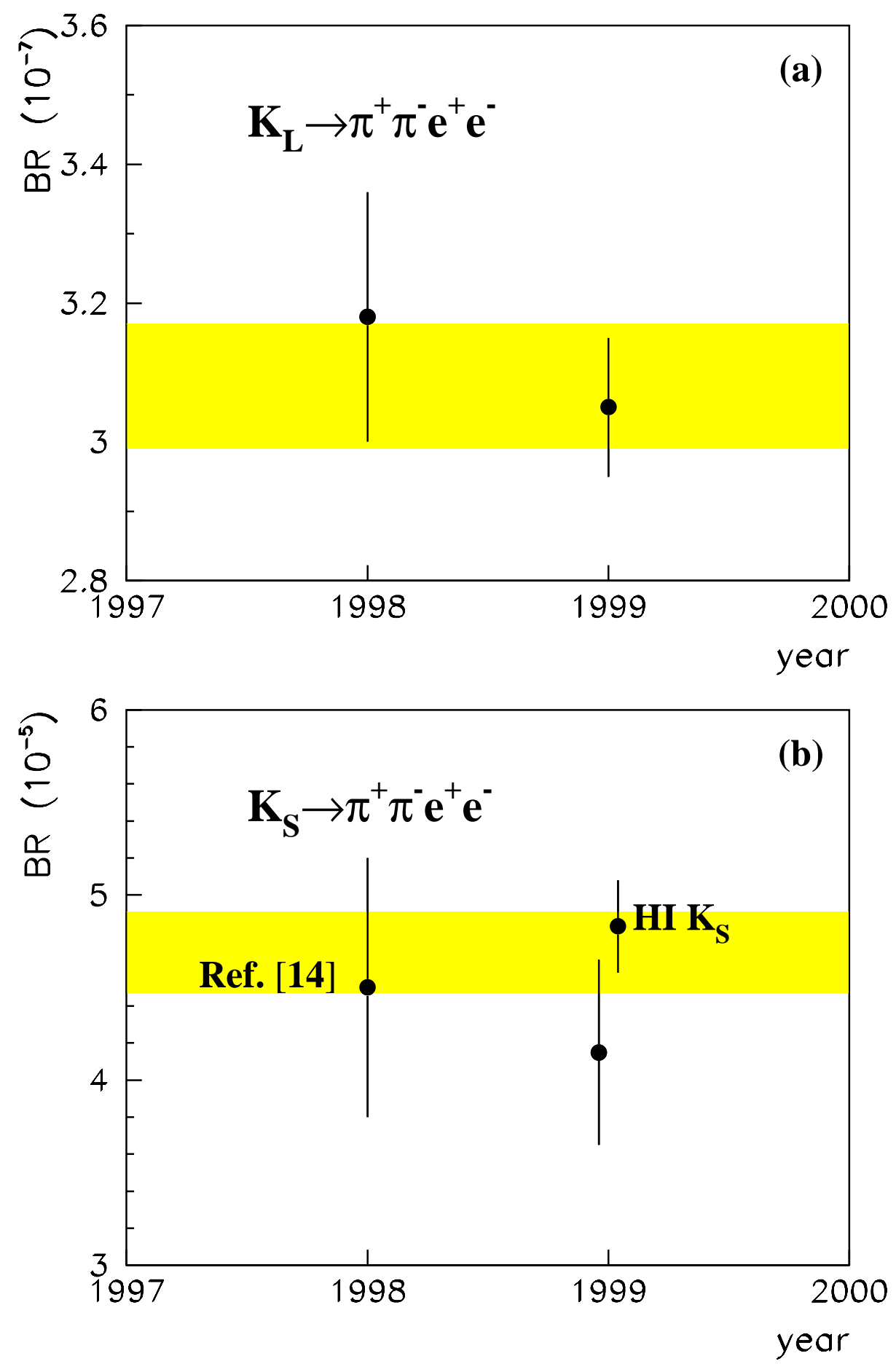

Figure 11: Branching ratio measurements as a function of run periods for $K_{\mathrm{L}} \rightarrow \pi^{+} \pi^{-} e^{+} e^{-}$ decays (a) and $K_{\mathrm{S}} \rightarrow \pi^{+} \pi^{-} e^{+} e^{-}$decays (b). The error bars reflect the statistical uncertainties only. The grey horiziontal bands represent the averaged values and the corresponding statistical uncertainties. 

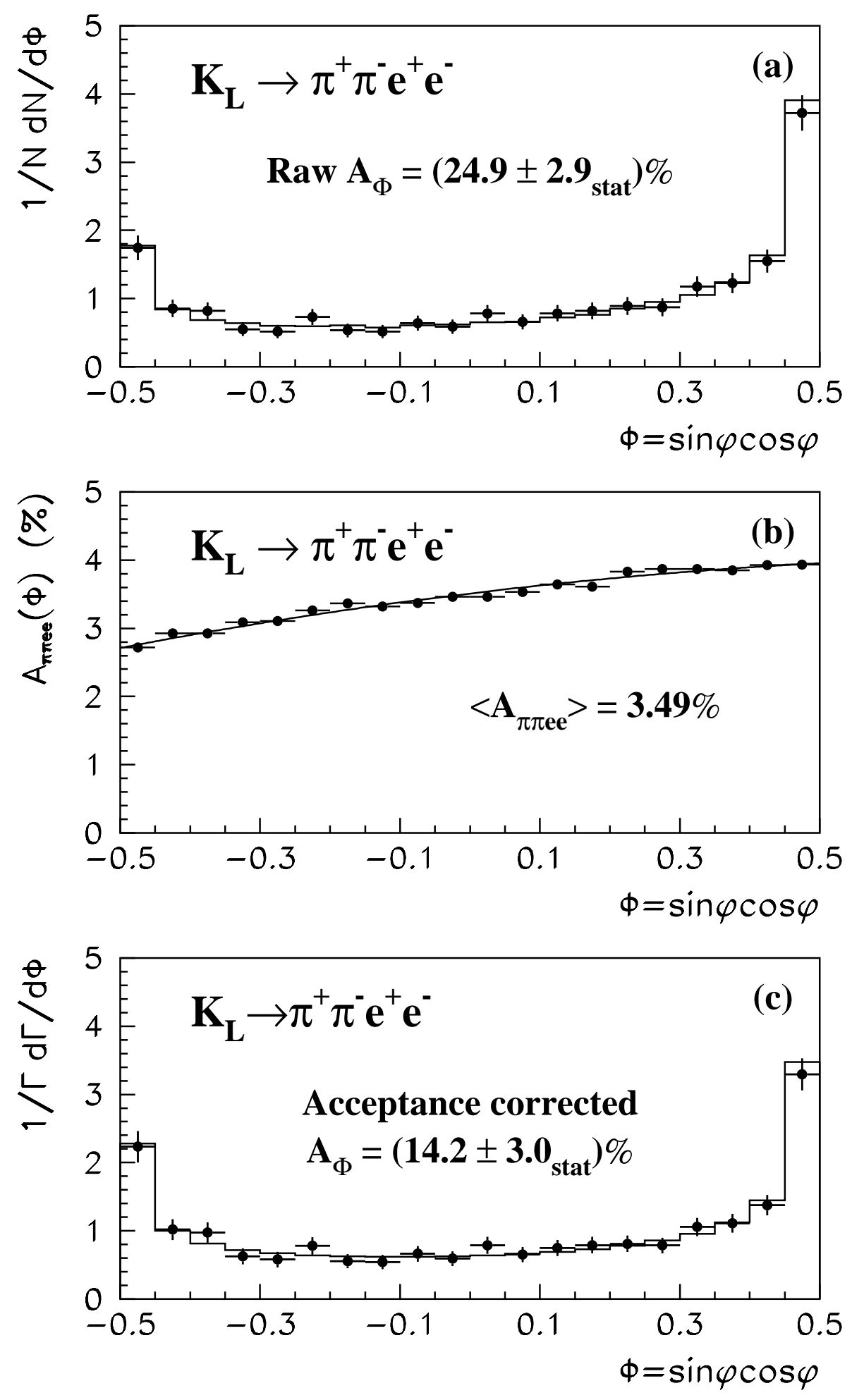

Figure 12: Distribution of $K_{\mathrm{L}} \rightarrow \pi^{+} \pi^{-} e^{+} e^{-}$events in the angular variable $\sin \phi \cos \phi$ before (a) and after (c) acceptance correction. The histograms are Monte Carlo predictions. Acceptance for $K_{\mathrm{L}} \rightarrow \pi^{+} \pi^{-} e^{+} e^{-}$decays as a function of $\sin \phi \cos \phi(\mathrm{b})$. The solid line is a polynomial fit to the Monte Carlo calculation. 

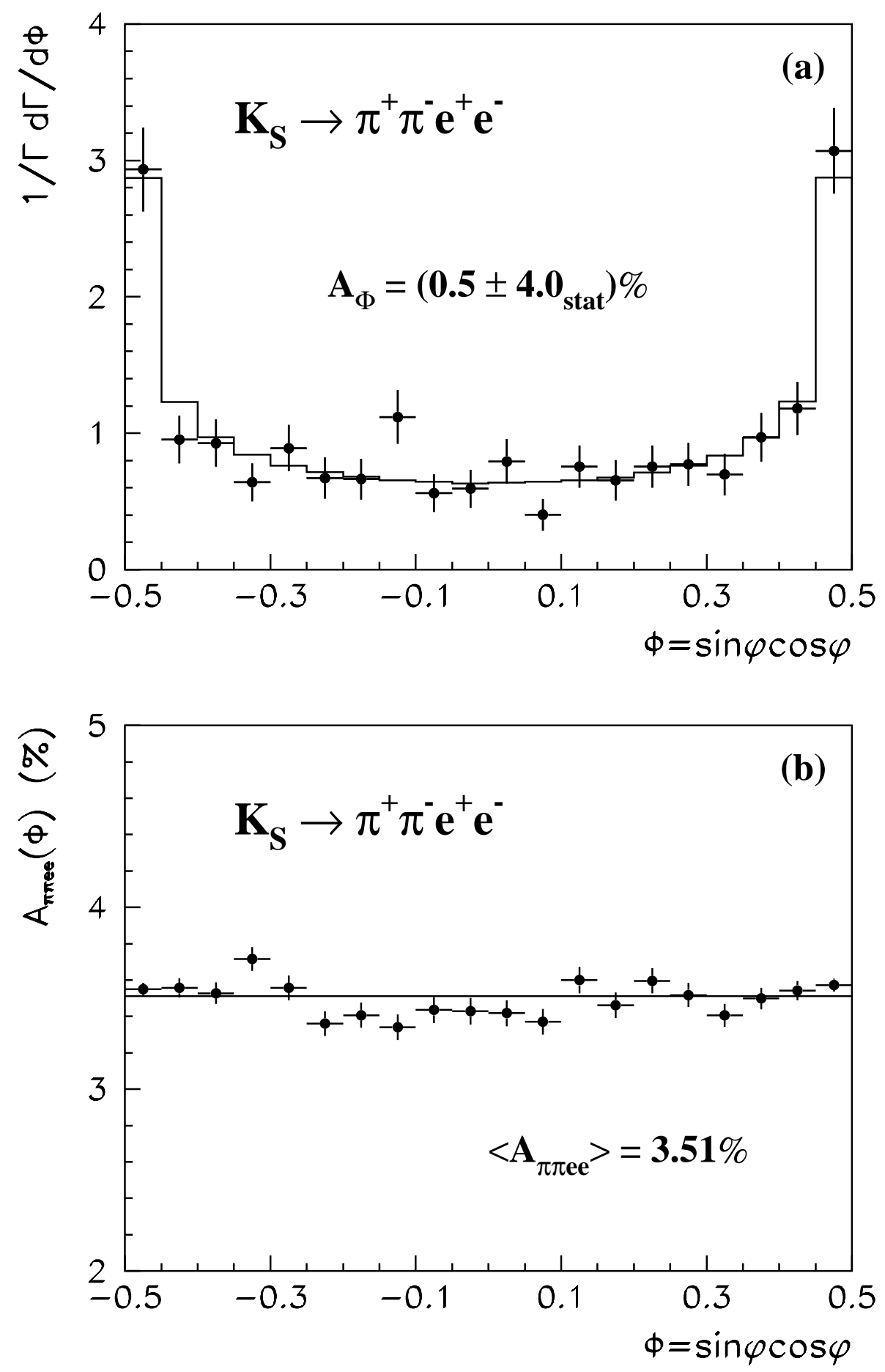

Figure 13: Distribution of events (a) and acceptance (b) for $K_{\mathrm{S}} \rightarrow \pi^{+} \pi^{-} e^{+} e^{-}$decays as a function of $\sin \phi \cos \phi$. The histogram in (a) is the Monte Carlo prediction. The solid line in (b) represents the average value of the acceptance. 